\title{
Knot Floer homology detects fibred knots
}

\author{
YI NI \\ Department of Mathematics, Princeton University \\ Princeton, New Jersey 08544, USA \\ Current address: Department of Mathematics, Columbia University \\ Room 516, MC 4406, 2990 Broadway \\ New York, NY 10027, USA \\ Email: yni@math.columbia.edu
}

\begin{abstract}
Ozsváth and Szabó conjectured that knot Floer homology detects fibred knots in $S^{3}$. We will prove this conjecture for null-homologous knots in arbitrary closed 3-manifolds. Namely, if $K$ is a knot in a closed 3 -manifold $Y, Y-K$ is irreducible, and $\widehat{H F K}(Y, K)$ is monic, then $K$ is fibred. The proof relies on previous works due to Gabai, Ozsváth-Szabó, Ghiggini and the author. A corollary is that if a knot in $S^{3}$ admits a lens space surgery, then the knot is fibred.
\end{abstract}

AMS Classification 57R58, 57M27; 57R30.

Keywords knot Floer homology, fibred knots, sutured manifold decomposition, lens space surgery.

Dedicated to Professor Boju Jiang on the occasion of his 70th birthday

\section{Introduction}

Knot Floer homology was introduced independently by Ozsváth-Szabó [13 and by Rasmussen [19]. For any null-homologous knot $K \subset Y^{3}$ with Seifert surface $F$, one can associate to it some abelian groups $\widehat{H F K}(Y, K,[F], i)$ for $i \in \mathbb{Z}$. The knot Floer homology

$$
\widehat{H F K}(Y, K) \cong \oplus_{i \in \mathbb{Z}} \widehat{H F K}(Y, K,[F], i)
$$

is a finitely generated abelian group.

A lot of topological information of the knot are contained in knot Floer homology, in particular in the topmost filtration level. For example, Ozsváth and Szabó proved that the topmost filtration level of $\widehat{H F K}$ for a knot in $S^{3}$ is exactly the genus of the knot (see [14]).

When $K$ is a fibred knot, it is shown that the topmost group of $\widehat{H F K}(Y, K)$ is a single $\mathbb{Z}[16]$. Ozsváth and Szabó conjectured that the converse is also true for knots in $S^{3}[18$. 
In this paper, we are going to prove this conjecture. Our main theorem is:

Theorem 1.1 Suppose $K$ is a null-homologous knot in a closed, oriented, connected 3-manifold $Y, Y-K$ is irreducible, and $F$ is a genus $g$ Seifert surface of $K$. If $\widehat{H F K}(Y, K,[F], g) \cong \mathbb{Z}$, then $K$ is fibred, and $F$ is a fibre of the fibration.

An oriented link $L$ in $Y$ is called a fibred link, if $Y-L$ fibres over the circle, and $L$ is the oriented boundary of the fibre. We have the following corollary of Theorem 1.1;

Corollary 1.2 Suppose $Y$ is a closed, oriented, connected 3-manifold, $L$ is a null-homologous oriented link in $Y, Y-L$ is irreducible, and $F$ is a Seifert surface of $L$. If $\widehat{H F K}\left(Y, L, \frac{|L|-\chi(F)}{2}\right) \cong \mathbb{Z}$, then $L$ is a fibred link, and $F$ is a fibre of the fibration.

The proof of this corollary will be given in Section 7 .

A rational homology sphere $Y$ is called an $L$-space, if the rank of $\widehat{H F}(Y)$ is equal to $\left|\mathrm{H}_{1}(Y ; \mathbb{Z})\right|$. Many 3 -manifolds are $L$-spaces, for example, the manifolds which admit spherical structures are $L$-spaces. An immediate corollary of Theorem 1.1 is:

Corollary 1.3 If a knot $K \subset S^{3}$ admits an $L$-space surgery, then $K$ is a fibred knot. In particular, any knot that admits a lens space surgery is fibred.

Proof As a corollary of [17, Proposition 9.5], if a rational surgery on $K$ yields an $L$-space, then $K$ also admits an $L$-space surgery with integer coefficient. Using [15, Theorem 1.2], we conclude that $\widehat{H F K}(K, g) \cong \mathbb{Z}$. Thus the desired result follows from Theorem 1.1 .

Corollary 1.4 Suppose $Y$ is an $L$-space, $K \subset Y$ is a null-homologous knot with genus $g>1$. If the 0 -surgery on $K$ is a surface bundle over $S^{1}$, then $K$ is fibred.

Proof With the above conditions, one can prove that

$$
H F^{+}\left(Y_{0}(K),[g-1]\right) \cong \widehat{H F K}(Y, K, g) .
$$

In fact, the proof is exactly the same as the proof of [13, Corollary 4.5], so we will not give the details. The reader should note that, since $Y$ is an $L$-space, 
$H F^{+}(Y)$ is isomorphic to the direct sum of some copies of $\mathbb{Z}\left[U, U^{-1}\right] / U \mathbb{Z}[U]$, hence the map $\psi$ as in the proof of [13, Corollary 4.5] is surjective. Thus the argument there can be used.

Now since $Y_{0}(K)$ fibres over the circle, we have $H F^{+}\left(Y_{0}(K),[g-1]\right) \cong \mathbb{Z}$, so $\widehat{H F K}(Y, K, g) \cong \mathbb{Z}$. It is easy to show that $Y-K$ is irreducible, hence $K$ is fibred by Theorem 1.1 .

Remark 1.5 The homology class $[F]$ defines a homomorphism

$$
f: \pi_{1}(Y-K) \rightarrow \mathbb{Z}
$$

by counting the intersection numbers of $[F]$ with loops. The famous Stallings' Fibration Theorem [20] says that $K$ is a fibred knot with fibre in the homology class of $[F]$ if and only if ker $f$ is finitely generated. Hence Theorem 1.1 indicates a mysterious relationship between Heegaard Floer homology and the fundamental group.

Theorem 1.1 was previously examined in various sporadic cases, and some theoretical evidences were given in [12, but the first real progress was made by Ghiggini in [5], where a strategy to approach this conjecture was proposed, and the special case of genus-one knots in $S^{3}$ was proved. Ghiggini's strategy plays an essential role in the present paper, we will apply this strategy by using a method introduced by Gabai [4. Another key ingredient of this paper is the study of decomposition formulas for knot Floer homology, which is based on 12 .

The paper is organized as follows. In Section 2, we give some backgrounds on sutured manifolds. We will introduce a sutured manifold invariant which naturally comes from knot Floer homology. We also present a construction of certain Heegaard diagrams. In Section 3, we prove a homological version of the main theorem. Section 4 is devoted to prove the horizontal decomposition formula for knot Floer homology. In Section 5, we will prove a major technical theorem: the decomposition formula for knot Floer homology, in the case of decomposing along a separating product annulus. In Section 6, we use Gabai's method to study Ghiggini's strategy. As a result, we get a clearer picture of the sutured manifold structure of the knot complement, namely, Theorem 6.2. Section 7 contains the proof of the main theorem, we use the decomposition formulas we proved (especially Theorem 5.1) to reduce the problem to the case that we already know.

Acknowledgements. This paper has been submitted to Princeton University as the author's PhD thesis. We wish to thank David Gabai and Zoltán Szabó for their guidance. 
We would like to thank Paolo Ghiggini for many fruitful discussions during the course of this work. This paper benefits a lot from his work [5].

A version of Theorem 6.2 was also proved by Ian Agol via a different approach. We wish to thank him for some interesting discussions.

We are grateful to Matthew Hedden, András Juhász, Tao Li, Peter Ozsváth, Jiajun Wang and Chenyang $\mathrm{Xu}$ for some helpful conversations and their interests in this work. We are particularly grateful to an anonymous referee for enormous suggestions and corrections.

The author was partially supported by a Graduate School Centennial Fellowship at Princeton University. Parts of the work were carried out when the author visited UQAM and Peking University; he wishes to thank Steve Boyer, Olivier Collin and Shicheng Wang for their hospitality. The author extends his gratitude to the American Institute of Mathematics and the Clay Mathematics Institute for their subsequent support.

\section{Preliminaries}

\subsection{Sutured manifold decomposition}

The theory of sutured manifold decomposition was introduced by Gabai in [2]. We will briefly review the basic definitions.

Definition 2.1 A sutured manifold $(M, \gamma)$ is a compact oriented 3-manifold $M$ together with a set $\gamma \subset \partial M$ of pairwise disjoint annuli $A(\gamma)$ and tori $T(\gamma)$. The core of each component of $A(\gamma)$ is a suture, and the set of sutures is denoted by $s(\gamma)$.

Every component of $R(\gamma)=\partial M-\operatorname{int}(\gamma)$ is oriented. Define $R_{+}(\gamma)$ (or $R_{-}(\gamma)$ ) to be the union of those components of $R(\gamma)$ whose normal vectors point out of (or into) $M$. The orientations on $R(\gamma)$ must be coherent with respect to $s(\gamma)$, hence every component of $A(\gamma)$ lies between a component of $R_{+}(\gamma)$ and a component of $R_{-}(\gamma)$.

Definition 2.2 [11, Definition 2.2] A balanced sutured manifold is a sutured manifold $(M, \gamma)$ satisfying

(1) $M$ has no closed components.

(2) Every component of $\partial M$ intersects $\gamma$ nontrivially.

(3) $\chi\left(R_{+}(\gamma)\right)=\chi\left(R_{-}(\gamma)\right)$. 
Definition 2.3 Let $S$ be a compact oriented surface with connected components $S_{1}, \ldots, S_{n}$. We define

$$
x(S)=\sum_{i} \max \left\{0,-\chi\left(S_{i}\right)\right\} .
$$

Let $M$ be a compact oriented 3-manifold, $A$ be a compact codimension-0 submanifold of $\partial M$. Let $h \in \mathrm{H}_{2}(M, A)$. The Thurston norm $x(h)$ of $h$ is defined to be the minimal value of $x(S)$, where $S$ runs over all the properly embedded surfaces in $M$ with $\partial S \subset A$ and $[S]=h$.

Definition 2.4 A sutured manifold $(M, \gamma)$ is taut, if $M$ is irreducible, and $R(\gamma)$ is Thurston norm minimizing in $\mathrm{H}_{2}(M, \gamma)$.

Definition 2.5 Let $(M, \gamma)$ be a sutured manifold, and $S$ a properly embedded surface in M, such that no component of $\partial S$ bounds a disk in $R(\gamma)$ and no component of $S$ is a disk with boundary in $R(\gamma)$. Suppose that for every component $\lambda$ of $S \cap \gamma$, one of (1)-(3) holds:

(1) $\lambda$ is a properly embedded non-separating arc in $\gamma$.

(2) $\lambda$ is a simple closed curve in an annular component $A$ of $\gamma$ in the same homology class as $A \cap s(\gamma)$.

(3) $\lambda$ is a homotopically nontrivial curve in a toral component $T$ of $\gamma$, and if $\delta$ is another component of $T \cap S$, then $\lambda$ and $\delta$ represent the same homology class in $\mathrm{H}_{1}(T)$.

Then $S$ is called a decomposition surface, and $S$ defines a sutured manifold decomposition

$$
(M, \gamma) \stackrel{S}{\rightsquigarrow}\left(M^{\prime}, \gamma^{\prime}\right)
$$

where $M^{\prime}=M-\operatorname{int}(\operatorname{Nd}(S))$ and

$$
\begin{aligned}
\gamma^{\prime} & =\left(\gamma \cap M^{\prime}\right) \cup \operatorname{Nd}\left(S_{+}^{\prime} \cap R_{-}(\gamma)\right) \cup \operatorname{Nd}\left(S_{-}^{\prime} \cap R_{+}(\gamma)\right), \\
R_{+}\left(\gamma^{\prime}\right) & =\left(\left(R_{+}(\gamma) \cap M^{\prime}\right) \cup S_{+}^{\prime}\right)-\operatorname{int}\left(\gamma^{\prime}\right), \\
R_{-}\left(\gamma^{\prime}\right) & =\left(\left(R_{-}(\gamma) \cap M^{\prime}\right) \cup S_{-}^{\prime}\right)-\operatorname{int}\left(\gamma^{\prime}\right),
\end{aligned}
$$

where $S_{+}^{\prime}\left(S_{-}^{\prime}\right)$ is that component of $\partial \mathrm{Nd}(S) \cap M^{\prime}$ whose normal vector points out of (into) $M^{\prime}$.

Definition 2.6 A decomposition surface is called a product disk, if it is a disk which intersects $s(\gamma)$ in exactly two points. A decomposition surface is called a product annulus, if it is an annulus with one boundary component in $R_{+}(\gamma)$, and the other boundary component in $R_{-}(\gamma)$. 
Definition 2.7 A decomposition surface $S$ in a balanced sutured manifold is called a horizontal surface, if $S$ has no closed component, $|\partial S|=|s(\gamma)|$, $[S]=\left[R_{+}(\gamma)\right] \in \mathrm{H}_{2}(M, \gamma)$, and $\chi(S)=\chi\left(R_{+}\right)$.

Definition 2.8 A balanced sutured manifold $(M, \gamma)$ is vertically prime, if any horizontal surface $S \subset M$ is parallel to either $R_{-}(\gamma)$ or $R_{+}(\gamma)$.

\subsection{Knot Floer homology and an invariant of sutured manifolds}

Heegaard Floer homology has been proved to have very close relationship with Thurston norm 14. The definition of Thurston norm is purely topological (or combinatorial) [21], while the definition of Heegaard Floer homology involves symplectic geometry and analysis. The bridge that connects these two seemingly different topics is taut foliation.

The fundamental method of constructing taut foliations is sutured manifold decomposition 2]. Thus we naturally expect that, by studying the behavior of Heegaard Floer homology under sutured manifold decomposition, we can get better understanding of the relationship between Heegaard Floer homology and Thurston norm.

The first approach in such direction was taken in [12, where the "sutured Heegaard diagrams" for knots are introduced. Using them, one can study a very special case of sutured manifold decomposition: the Murasugi sum. Later on, Juhász introduced an invariant for sutured manifolds, called "sutured Floer homology", and proved some properties [11].

In this subsection, we will introduce another invariant of sutured manifolds, which naturally comes from knot Floer homology. We will prove some decomposition formulas for this invariant in Section 4 and Section 5.

Suppose $L \subset Y$ is a null-homologous oriented link, $F$ is a Seifert surface of $L$. Decompose $Y$-int $(\operatorname{Nd}(L))$ along $F$, we get a balanced sutured manifold $(M, \gamma)$. The argument in [12, Proposition 3.5] shows that, if we cut open $Y$ along $F$, reglue by a homeomorphism of $F$ which is the identity on the boundary, to get a new link $L^{\prime}$ in a new manifold $Y^{\prime}$, then

$$
\widehat{H F K}\left(Y, L, \frac{|\partial F|-\chi(F)}{2}\right) \cong \widehat{H F K}\left(Y^{\prime}, L^{\prime}, \frac{|\partial F|-\chi(F)}{2}\right)
$$

as abelian groups. Therefore, $\widehat{H F K}\left(Y, L, \frac{|\partial F|-\chi(F)}{2}\right)$ can be viewed as an invariant for the sutured manifold $(M, \gamma)$. (For simplicity, let $\mathfrak{i}(F)$ denote $\frac{|\partial F|-\chi(F)}{2}$.) 
More precisely, suppose $(M, \gamma)$ is a balanced sutured manifold, $R_{ \pm}(\gamma)$ are connected surfaces. There exists a diffeomorphism

$$
\psi: R_{+}(\gamma) \rightarrow R_{-}(\gamma),
$$

such that for each component $A$ of $\gamma, \psi$ maps one boundary component of $A$ onto the other boundary component. We glue $R_{+}(\gamma)$ to $R_{-}(\gamma)$ by $\psi$, thus get a manifold with boundary consisting of tori. We fill each boundary torus by a solid torus whose meridian intersects $s(\gamma)$ exactly once. Now we get a closed 3 -manifold $Y$. Let $L$ be the union of the cores of the solid tori. The pair $(Y, L)$ is denoted by $\iota(M, \gamma)$. Of course, $\iota(M, \gamma)$ depends on the way we glue $R_{+}$to $R_{-}$and the way we fill in the solid tori. In our case, changing the filling is equivalent to changing the gluing map by Dehn twists along the components of $\partial R_{+}$. By the remark in the last paragraph, the abelian group

$$
\widehat{H F K}\left(\iota(M, \gamma), \mathfrak{i}\left(R_{+}(\gamma)\right)\right)
$$

is independent of the choice of the gluing, hence it is independent of the choice of $\iota(M, \gamma)$.

Proposition 2.9 There is a well-defined invariant $\widehat{H F S}$ for balanced sutured manifolds, which is characterized by the following two properties.

(1) If $(M, \gamma)$ is a balanced sutured manifold, $R_{ \pm}(\gamma)$ are connected, then

$$
\widehat{H F S}(M, \gamma) \cong \widehat{H F K}\left(\iota(M, \gamma), \mathfrak{i}\left(R_{+}(\gamma)\right)\right)
$$

(2) If

$$
(M, \gamma) \stackrel{a \times I}{\rightsquigarrow}\left(M^{\prime}, \gamma^{\prime}\right),
$$

is the decomposition along a product disk then

$$
\widehat{H F S}(M, \gamma) \cong \widehat{H F S}\left(M^{\prime}, \gamma^{\prime}\right) \text {. }
$$

Proof The inverse operation of the decomposition along a product disk, is "adding a product 1-handle" with feet at the suture. We first claim that, if $(M, \gamma)$ is a balanced sutured manifold with $R_{ \pm}(\gamma)$ connected, and $\left(M_{1}, \gamma_{1}\right)$ is obtained by adding a product 1 -handle to $(M, \gamma)$, then

$$
\widehat{H F K}\left(\iota(M, \gamma), \mathfrak{i}\left(R_{+}(\gamma)\right)\right) \cong \widehat{H F K}\left(\iota\left(M_{1}, \gamma_{1}\right), \mathfrak{i}\left(R_{+}\left(\gamma_{1}\right)\right)\right) .
$$

In fact, one possible choice of $\iota\left(M_{1}, \gamma_{1}\right)$ can be gotten by plumbing $\iota(M, \gamma)$ with $\left(S^{2} \times S^{1}, \Pi\right)$. Here $\Pi$ is a link in $S^{2} \times S^{1}$ which consists of two copies of point $\times S^{1}$, but with different orientations. Now we can apply [12, Lemma 4.4] to get the claim. 
Given a balanced sutured manifold $(M, \gamma)$, we can add to it some product 1handles with feet at the suture to get a new balanced sutured manifold $\left(M_{1}, \gamma_{1}\right)$, such that $R_{ \pm}\left(\gamma_{1}\right)$ are connected. We then define

$$
\widehat{H F S}(M, \gamma)=\widehat{H F K}\left(\iota\left(M_{1}, \gamma_{1}\right), \mathfrak{i}\left(R_{+}\left(\gamma_{1}\right)\right)\right) .
$$

Now we want to prove that $\widehat{H F S}(M, \gamma)$ is independent of the choice of $\left(M_{1}, \gamma_{1}\right)$. For this purpose, let $\left(M_{2}, \gamma_{2}\right)$ be another sutured manifold obtained by adding product 1 -handles to $(M, \gamma)$, and $R_{ \pm}\left(\gamma_{2}\right)$ are connected. We can assume that the feet of the product 1-handles for $\left(M_{1}, \gamma_{1}\right)$ and $\left(M_{2}, \gamma_{2}\right)$ are mutually different. Let $\left(M_{3}, \gamma_{3}\right)$ be the sutured manifold obtained by adding all these product 1-handles (either for $M_{1}$ or for $M_{2}$ ) to $(M, \gamma)$. By the claim proved in the first paragraph, we have

$$
\begin{aligned}
& \widehat{H F K}\left(\iota\left(M_{3}, \gamma_{3}\right), \mathfrak{i}\left(R_{+}\left(\gamma_{3}\right)\right)\right) \cong \widehat{H F K}\left(\iota\left(M_{1}, \gamma_{1}\right), \mathfrak{i}\left(R_{+}\left(\gamma_{1}\right)\right)\right), \\
& \widehat{H F K}\left(\iota\left(M_{3}, \gamma_{3}\right), \mathfrak{i}\left(R_{+}\left(\gamma_{3}\right)\right)\right) \cong \widehat{H F K}\left(\iota\left(M_{2}, \gamma_{2}\right), \mathfrak{i}\left(R_{+}\left(\gamma_{2}\right)\right)\right) .
\end{aligned}
$$

Therefore, $\widehat{H F S}(M, \gamma)$ is well-defined.

Property (1) holds by definition, and Property (2) can be proved by the same argument as above.

\subsection{Relative Morse functions and sutured diagrams}

Suppose $K$ is a null-homologous knot in $Y, F$ is a Seifert surface for $K$. In [12], the notion of "sutured Heegaard diagrams" was introduced. Such diagrams are useful to compute $\widehat{H F K}(Y, K,[F], g)$. A construction of sutured Heegaard diagrams was given in the proof of [12, Theorem 2.1].

In this subsection, we will present a slightly different construction, which is based on relative Morse functions. This construction will be useful later.

Definition 2.10 [12, Definition 2.2] A double pointed Heegaard diagram

$$
\left(\Sigma, \boldsymbol{\alpha}, \boldsymbol{\beta}_{\mathbf{0}} \cup\{\mu\}, w, z\right)
$$

for $(Y, K)$ is a sutured Heegaard diagram, if it satisfies:

(Su0) There exists a subsurface $\mathcal{P} \subset \Sigma$, bounded by two curves $\alpha_{1} \in \boldsymbol{\alpha}$ and $\lambda$. $g$ denotes the genus of $\mathcal{P}$.

(Su1) $\lambda$ is disjoint from $\boldsymbol{\beta}_{\mathbf{0}} \cdot \boldsymbol{\mu}$ does not intersect any $\alpha$ curves except $\alpha_{1} \cdot \mu$ intersects $\lambda$ transversely in exactly one point, and intersects $\alpha_{1}$ transversely 
in exactly one point. $w, z \in \lambda$ lie in a small neighborhood of $\lambda \cap \mu$, and on different sides of $\mu$. (In practice, we often push $w, z$ off $\lambda$ into $\mathcal{P}$ or $\Sigma-\mathcal{P}$.)

(Su2) $\left(\boldsymbol{\alpha}-\left\{\alpha_{1}\right\}\right) \cap \mathcal{P}$ consists of $2 g$ arcs, which are linearly independent in $\mathrm{H}_{1}(\mathcal{P}, \partial \mathcal{P})$. Moreover, $\Sigma-\boldsymbol{\alpha}-\mathcal{P}$ is connected.

Construction 2.11 Suppose $(M, \gamma)$ is the sutured manifold obtained by cutting $Y-\operatorname{int}(\operatorname{Nd}(K))$ open along $F$. Let $\psi: R_{+}(\gamma) \rightarrow R_{-}(\gamma)$ be the gluing map. Namely, if we glue $R_{+}(\gamma)$ to $R_{-}(\gamma)$ by $\psi$, then we get back the manifold $Y-\operatorname{int}(\operatorname{Nd}(K))$. We will construct a Heegaard diagram for the pair $(Y, K)$. The construction consists of 4 steps.

Step 0. A relative Morse function

Consider a self-indexed relative Morse function $u$ on $(M, \gamma)$. Namely, $u$ satisfies:

(1) $u(M)=[0,3], u^{-1}(0)=R_{-}(\gamma), u^{-1}(3)=R_{+}(\gamma)$.

(2) $u$ has no degenerate critical points. $u$ is the standard height function near $\gamma \cdot u^{-1}\{$ critical points of index $i\}=i$.

(3) $u$ has no critical points on $R(\gamma)$.

Let $\widetilde{F}=u^{-1}\left(\frac{3}{2}\right) . \partial \widetilde{F}$ is denoted by $\widetilde{\lambda}$. Similarly, the boundary components of $R_{ \pm}(\gamma)$ are denoted by $\lambda_{ \pm}$.

Suppose $u$ has $r$ index -1 critical points, then the genus of $\widetilde{F}$ is $g+r$. The gradient $-\nabla u$ generates a flow $\phi_{t}$ on $M$. There are $2 r$ points on $R_{+}(\gamma)$, which are connected to index -2 critical points by flowlines. We call these points "bad" points. Similarly, there are $2 r$ bad points on $R_{-}(\gamma)$, which are connected to index -1 critical points by flowlines.

Step 1. Construct the curves

Choose a small disk $D_{+}$in a neighborhood of $\lambda_{+}$in $R_{+}(\gamma)$. Choose an arc $\delta_{+} \subset R_{+}(\gamma)$ connecting $D_{+}$to $\lambda_{+}$. Flow $D_{+}$and $\delta_{+}$by $\phi_{t}$, their images on $\widetilde{F}$ and $R_{-}(\gamma)$ are $\widetilde{D}, D_{-}, \widetilde{\delta}, \delta_{-}$. (Of course, we choose $D_{+}$and $\delta_{+}$generically, so that the flowlines starting from them do not terminate at critical points.) We can suppose the gluing map $\psi$ maps $\delta_{+}$onto $\delta_{-}, D_{+}$onto $D_{-}$. Let $A_{ \pm}=R_{ \pm}(\gamma)-\operatorname{int}\left(D_{ \pm}\right), \widetilde{A}=\widetilde{F}-\operatorname{int}(\widetilde{D})$.

On $\widetilde{F}$, there are $r$ simple closed curves $\widetilde{\alpha}_{2 g+2}, \ldots, \widetilde{\alpha}_{2 g+1+r}$, which are connected to index -1 critical points by flowlines. And there are $r$ simple closed curves $\widetilde{\beta}_{2 g+2}, \ldots, \widetilde{\beta}_{2 g+1+r}$, which are connected to index -2 critical points by flowlines. 
Choose $2 g$ disjoint $\operatorname{arcs} \xi_{2}^{-}, \ldots, \xi_{2 g+1}^{-} \subset A_{-}$, such that their endpoints lie on $\lambda_{-}$, and they are linearly independent in $\mathrm{H}_{1}\left(A_{-}, \partial A_{-}\right)$. We also suppose they are disjoint from $\delta_{-}$and the bad points. Let $\xi_{i}^{+}=\psi^{-1}\left(\xi_{i}^{-}\right)$. We also flow back $\xi_{2}^{-}, \ldots, \xi_{2 g+1}^{-}$by $\phi_{-t}$ to $\widetilde{F}$, the images are denoted by $\widetilde{\xi}_{2}, \ldots, \widetilde{\xi}_{2 g+1}$.

Choose $2 g$ disjoint $\operatorname{arcs} \eta_{2}^{+}, \ldots, \eta_{2 g+1}^{+} \subset A_{+}$, such that their endpoints lie on $\partial D_{+}$, and they are linearly independent in $\mathrm{H}_{1}\left(A_{+}, \partial A_{+}\right)$. We also suppose they are disjoint from $\delta_{+}$and the bad points. Flow them by $\phi_{t}$ to $\widetilde{F}$, the images are denoted by $\widetilde{\eta}_{2}, \ldots, \widetilde{\eta}_{2 g+1}$.

\section{Step 2. Construct a diagram}

Let

$$
\Sigma=A_{+} \cup \widetilde{A} \cup\{2 \text { annuli }\}
$$

where one annulus is $\phi_{[0,3 / 2]}\left(\partial D_{+}\right)$, the other is $\phi_{[0,3 / 2]}(\widetilde{\lambda})$. Let

$$
\alpha_{i}=\xi_{i}^{+} \cup \widetilde{\xi}_{i} \cup\{2 \operatorname{arcs}\},
$$

where the 2 arcs are vertical arcs connecting $\xi_{i}^{+}$to $\widetilde{\xi}_{i}$ on an annulus, $i=$ $2, \ldots, 2 g+1$. Similarly, let

$$
\beta_{i}=\eta_{i}^{+} \cup \widetilde{\eta}_{i} \cup\{2 \operatorname{arcs}\} .
$$

Let $\alpha_{1}=\partial \widetilde{D}, \lambda=\widetilde{\lambda}$

$$
\mu=\delta_{+} \cup \widetilde{\delta} \cup\{2 \operatorname{arcs}\}
$$

Let

$$
\begin{aligned}
\boldsymbol{\alpha} & =\left\{\alpha_{2}, \ldots, \alpha_{2 g+1}\right\} \cup\left\{\widetilde{\alpha}_{2 g+2}, \ldots, \widetilde{\alpha}_{2 g+1+r}\right\} \cup\left\{\alpha_{1}\right\}, \\
\boldsymbol{\beta} & =\left\{\beta_{2}, \ldots, \beta_{2 g+1}\right\} \cup\left\{\widetilde{\beta}_{2 g+2}, \ldots, \widetilde{\beta}_{2 g+1+r}\right\} \cup\{\mu\} .
\end{aligned}
$$

Pick two base points $w, z$ near $\lambda \cap \mu$, but on different sides of $\mu$.

Step 3. Check that $(\Sigma, \boldsymbol{\alpha}, \boldsymbol{\beta}, w, z)$ is a Heegaard diagram for $(Y, K)$

This step is quite routine, we leave the reader to check the following

(A) $\Sigma$ separates $Y$ into two genus- $(2 g+1+r)$ handlebodies $U_{1}, U_{2}$, where

$$
U_{1}=u^{-1}\left[0, \frac{3}{2}\right] \bigcup \phi_{\left[0, \frac{3}{2}\right]}\left(D_{+}\right), \quad U_{2}=u^{-1}\left[\frac{3}{2}, 3\right]-\phi_{\left[0, \frac{3}{2}\right]}\left(D_{+}\right) .
$$

Every curve in $\boldsymbol{\alpha}$ bounds a disk in $U_{1}$, every curve in $\boldsymbol{\beta}$ bounds a disk in $U_{2}$.

(B) $\Sigma-\boldsymbol{\alpha}$ is connected, $\Sigma-\boldsymbol{\beta}$ is connected.

(C) The two base points $w, z$ give the knot $K$ in $Y$. 
Then

$$
(\Sigma, \boldsymbol{\alpha}, \boldsymbol{\beta}, w, z)
$$

is a Heegaard diagram for $(Y, K)$. It is not hard to see that this is a sutured Heegaard diagram.

The idea of considering a relative Morse function on the sutured manifold appeared in [6]. This idea was communicated to the author by Zoltán Szabó, and was used by András Juhász to define a sutured manifold invariant [11].

Lemma 2.12 We can wind the $\widetilde{\alpha}$-curves in $\widetilde{A}-\widetilde{\delta}$, and wind the $\xi^{+}$-curves in $A_{+}-\delta_{+}$, to get a weakly admissible Heegaard diagram.

Proof We claim that,

$$
E=\widetilde{A}-\cup_{i=2}^{2 g+1} \widetilde{\xi}_{i}-\cup_{j=2 g+2}^{2 g+1+r} \widetilde{\alpha}_{j}-\widetilde{\delta}
$$

is connected. In fact, since $A_{-}$is connected, and $\xi_{j}^{-}$'s are linearly independent in $\mathrm{H}_{1}\left(A_{-}, \partial A_{-}\right)$, it is easy to see that $E_{-}=A_{-}-\cup_{i=2}^{2 g+1} \xi_{i}^{-}-\delta_{-}$is connected. $E$ is obtained by removing $2 r$ disks from $E_{-}$, so $E$ is also connected.

Since $E$ is connected, we can find simple closed curves

$$
\theta_{2 g+2}, \ldots, \theta_{2 g+1+r} \subset \widetilde{A}-\cup \widetilde{\xi}_{i}-\widetilde{\delta}
$$

which are geometrically dual to $\widetilde{\alpha}_{2 g+2}, \ldots, \widetilde{\alpha}_{2 g+1+r}$. Namely, $\theta_{j}$ is disjoint from $\widetilde{\alpha}_{i}$ when $j \neq i$, and $\theta_{i}$ intersects $\widetilde{\alpha}_{i}$ transversely at exactly one point.

We can also find closed curves in $A_{+}-\delta_{+}$, which are geometrically dual to the $\xi^{+}$-curves. Now our desired result follows from the argument in [12, Proposition $3.3]$.

\section{A homological version of the main theorem}

Proposition 3.1 Suppose $K \subset Y$ is a null-homologous knot with Seifert surface $F,(M, \gamma)$ is the sutured manifold obtained by cutting $Y-\operatorname{int}(\operatorname{Nd}(K))$ open along $F$.

If $\widehat{H F K}(Y, K,[F], g) \cong \mathbb{Z}$, then $M$ is a homology product, namely,

$$
\mathrm{H}_{*}\left(M, R_{-}(\gamma) ; \mathbb{Z}\right) \cong \mathrm{H}_{*}\left(M, R_{+}(\gamma) ; \mathbb{Z}\right) \cong 0 .
$$

Fix a field $\mathbb{F}$, let $0 \in \mathbb{F}$ be the zero element. 
Lemma 3.2 If $\widehat{H F K}(Y, K,[F], g) \cong \mathbb{Z}$, then $\mathrm{H}_{2}(M ; \mathbb{F})=0$.

Proof We use the sutured diagram constructed in Construction 2.11. For the generators of $\widehat{H F K}(Y, K,[F],-g)$, the intersection point $\mu \cap \alpha_{1}$ is always chosen. The generators are supported in $\widetilde{A}[13$, Theorem 5.1], hence the determinant of the $(2 g+r) \times(2 g+r)$ matrix

$$
V=\left(\begin{array}{cc}
\widetilde{\xi}_{i} \cdot \widetilde{\eta}_{j} & \widetilde{\xi}_{i} \cdot \widetilde{\beta}_{l} \\
\widetilde{\alpha}_{k} \cdot \widetilde{\eta}_{j} & \widetilde{\alpha}_{k} \cdot \widetilde{\beta}_{l}
\end{array}\right) \quad \begin{aligned}
& 2 \leq i, j \leq 2 g+1 \\
& 2 g+2 \leq k, l \leq 2 g+1+r
\end{aligned}
$$

computes the Euler characteristic of $\widehat{H F K}(Y, K,[F], g) \cong \mathbb{Z}$. Hence

$$
\operatorname{det} V= \pm 1 \text {. }
$$

Now if $\mathrm{H}_{2}(M ; \mathbb{F}) \neq 0$, then there is a nontrivial $\mathbb{F}$-linear combination

$$
\gamma=\sum a_{k} \widetilde{\alpha}_{k}+\sum b_{l} \widetilde{\beta}_{l}
$$

which is 0 in $\mathrm{H}_{1}(\widetilde{F} ; \mathbb{F})$. So $\gamma \cdot \widetilde{\alpha}_{k}=0, \gamma \cdot \widetilde{\xi}_{i}=0$.

Since $\widetilde{\alpha}$-curves are linearly independent in $\mathrm{H}_{1}(\widetilde{\Sigma} ; \mathbb{F})$, the coefficients $b_{l}$ 's are not all 0 . Without loss of generality, we can assume $b_{2 g+2} \neq 0$.

Since the $\widetilde{\alpha}$-curves and $\widetilde{\xi}$-curves are mutually disjoint, we have

$$
\widetilde{\alpha}_{k} \cdot \sum b_{l} \widetilde{\beta}_{l}=0, \quad \widetilde{\xi}_{i} \cdot \sum b_{l} \widetilde{\beta}_{l}=0 .
$$

So by elementary column operations, we can change $V$ into a matrix with a zero column, while the determinant of this new matrix is $b_{2 g+2} \operatorname{det} V \neq 0$. This gives a contradiction.

Lemma 3.3 If $\widehat{H F K}(Y, K,[F], g) \cong \mathbb{Z}$, then the map

$$
i_{*}: \mathrm{H}_{1}\left(R_{-}(\gamma), \partial R_{-}(\gamma) ; \mathbb{F}\right) \rightarrow \mathrm{H}_{1}(M, \gamma ; \mathbb{F})
$$

is injective.

Proof $\mathrm{H}_{1}\left(R_{-}(\gamma), \partial R_{-}(\gamma)\right)$ is generated by the $\xi^{-}$-curves. If $i_{*}$ is not injective, then there exists a nontrivial linear combination

$$
\zeta=\sum c_{i} \widetilde{\xi}_{i}
$$

which is homologous to a linear combination

$$
\gamma=\sum a_{k} \widetilde{\alpha}_{k}+\sum b_{l} \widetilde{\beta}_{l}
$$


in $\mathrm{H}_{1}(\widetilde{F}, \partial \widetilde{F} ; \mathbb{F})$. We have $(\zeta-\gamma) \cdot \widetilde{\beta}_{l}=0$. The $\widetilde{\eta}$-curves are non-proper arcs in $\widetilde{F}$, but we can connect the two endpoints of $\widetilde{\eta}_{j}$ by an arc in $\widetilde{D}$ to get a closed curve in $\widetilde{F} . \widetilde{\xi}_{i}, \widetilde{\alpha}_{k}, \widetilde{\beta}_{l}$ do not intersect $\widetilde{D}$, so $(\zeta-\gamma) \cdot \widetilde{\eta}_{j}=0$. Since $\widetilde{\beta}$-curves and $\widetilde{\eta}$-curves are mutually disjoint, we have

$$
\widetilde{\eta}_{j} \cdot\left(\zeta-\sum a_{k} \widetilde{\alpha}_{k}\right)=0, \quad \widetilde{\beta}_{l} \cdot\left(\zeta-\sum a_{k} \widetilde{\alpha}_{k}\right)=0
$$

Now we can get a contradiction as in the proof of Lemma 3.2 ,

Proof of Proposition 3.1 Let $R_{ \pm}=R_{ \pm}(\gamma)$. By Lemma 3.3, we have the exact sequence

$$
0 \rightarrow \mathrm{H}_{2}\left(R_{-}, \partial R_{-} ; \mathbb{F}\right) \rightarrow \mathrm{H}_{2}\left(M, \partial R_{-} ; \mathbb{F}\right) \rightarrow \mathrm{H}_{2}\left(M, R_{-} ; \mathbb{F}\right) \rightarrow 0 .
$$

Compare this exact sequence with

$$
\mathrm{H}_{2}(M ; \mathbb{F}) \rightarrow \mathrm{H}_{2}(M, \gamma ; \mathbb{F}) \rightarrow \mathrm{H}_{1}(\gamma ; \mathbb{F}) \rightarrow \cdots .
$$

Note that

$$
\mathrm{H}_{2}(M, \gamma)=\mathrm{H}_{2}\left(M, \partial R_{-}\right), \quad \mathrm{H}_{2}\left(R_{-}, \partial R_{-}\right) \cong \mathrm{H}_{1}(\gamma) \cong \mathbb{Z},
$$

and $\mathrm{H}_{2}(M ; \mathbb{F})=0$ by Lemma $[3.2$, so

$$
\mathrm{H}_{2}\left(M, R_{-} ; \mathbb{F}\right)=0 \text {. }
$$

By Poincaré duality, we have

$$
\mathrm{H}_{1}(M, \partial M ; \mathbb{F}) \cong \mathrm{H}^{2}(M ; \mathbb{F}) \cong \mathrm{H}_{2}(M ; \mathbb{F}) \cong 0 .
$$

So we have the exact sequence

$$
0 \rightarrow \mathrm{H}_{2}(M, \partial M ; \mathbb{F}) \rightarrow \mathrm{H}_{1}\left(\partial M, R_{-} ; \mathbb{F}\right) \rightarrow \mathrm{H}_{1}\left(M, R_{-} ; \mathbb{F}\right) \rightarrow 0 .
$$

Compare this exact sequence with

$$
0 \rightarrow \mathrm{H}_{1}\left(R_{-} ; \mathbb{F}\right) \rightarrow \mathrm{H}_{1}(M ; \mathbb{F}) \rightarrow \mathrm{H}_{1}\left(M, R_{-} ; \mathbb{F}\right) \rightarrow 0 .
$$

Note that

$$
\begin{gathered}
\mathrm{H}_{1}\left(\partial M, R_{-} ; \mathbb{F}\right) \cong \mathrm{H}_{1}\left(R_{-} ; \mathbb{F}\right) \cong \mathbb{F}^{2 g}, \\
\mathrm{H}_{1}(M ; \mathbb{F}) \cong \mathrm{H}^{1}(M ; \mathbb{F}) \cong \mathrm{H}_{2}(M, \partial M ; \mathbb{F}),
\end{gathered}
$$

we should have

$$
\mathrm{H}_{1}\left(M, R_{-} ; \mathbb{F}\right)=0 .
$$

Hence we have proved that $\mathrm{H}_{*}\left(M, R_{-} ; \mathbb{F}\right)=0$ for any field $\mathbb{F}$. So

$$
\mathrm{H}_{*}\left(M, R_{-} ; \mathbb{Z}\right)=0 .
$$

Similarly, we have $\mathrm{H}_{*}\left(M, R_{+} ; \mathbb{Z}\right)=0$. 


\section{Horizontal decomposition}

Theorem 4.1 Let $K^{\prime} \subset Y^{\prime}, K^{\prime \prime} \subset Y^{\prime \prime}$ be two null-homologous knots. Suppose $F^{\prime}, F^{\prime \prime}$ are two genus-g Seifert surfaces for $K^{\prime}, K^{\prime \prime}$, respectively. We construct a new manifold $Y$ and a knot $K \subset Y$ as follows. Cut open $Y^{\prime}, Y^{\prime \prime}$ along $F^{\prime}, F^{\prime \prime}$, we get sutured manifolds $\left(M^{\prime}, \gamma^{\prime}\right)$, $\left(M^{\prime \prime}, \gamma^{\prime \prime}\right)$. Now glue $R_{+}\left(\gamma^{\prime}\right)$ to $R_{-}\left(\gamma^{\prime \prime}\right)$, glue $R_{+}\left(\gamma^{\prime \prime}\right)$ to $R_{-}\left(\gamma^{\prime}\right)$, by two diffeomorphisms. We get a manifold $Z$ with torus boundary. There is a simple closed curve $\mu \subset \partial Z$, which is the union of the two cut-open meridians of $K^{\prime}, K^{\prime \prime}$. We do Dehn filling along $\mu$ to get the manifold $Y$, the knot $K$ is the core of the filled-in solid torus.

Our conclusion is

$$
\widehat{H F K}\left(Y, K,\left[F^{\prime}\right], g\right) \cong \widehat{H F K}\left(Y^{\prime}, K^{\prime},\left[F^{\prime}\right], g\right) \otimes \widehat{H F K}\left(Y^{\prime \prime}, K^{\prime \prime},\left[F^{\prime \prime}\right], g\right),
$$

as linear spaces over any field $\mathbb{F}$.

Remark 4.2 We did not specify the gluings, since they will not affect our result, thanks to [12, Proposition 3.5].

Remark 4.3 We clarify some convention we are going to use throughout this paper. A holomorphic disk in the symmetric product is seen as an immersed subsurface of the Heegaard surface $\Sigma$. Suppose $Q$ is a subsurface of $\Sigma, D_{1}, \ldots, D_{n}$ are the closures of the components of $Q-\cup \alpha_{i}-\cup \beta_{j}$, choose a point $z_{k}$ in the interior of $D_{k}$ for each $k$. If $\Phi$ is a holomorphic disk, then $\Phi \cap Q$ denotes the immersed surface $\sum_{k} n_{z_{k}}(\Phi) D_{k}$.

Proof of Theorem 4.1 The proof uses the techniques from [12]. We construct a sutured Heegaard diagram $\left(\Sigma^{\prime}, \boldsymbol{\alpha}^{\prime}, \boldsymbol{\beta}^{\prime}, w^{\prime}, z^{\prime}\right)$ for $\left(Y^{\prime}, K^{\prime}\right)$, as in the proof of [12, Theorem 2.1]. The reader may refer to Figure 1 there for a partial picture.

As a result, $\Sigma^{\prime}$ is the union of two compact surfaces $A^{\prime}, B^{\prime}$, where $A^{\prime}$ is a genus $g$ surface with two boundary components $\alpha_{1 A}^{\prime}, \lambda_{A}^{\prime}$, and $B^{\prime}$ is a genus $g+r^{\prime}$ surface with two boundary components $\alpha_{1 B}^{\prime}, \lambda_{B}^{\prime} \cdot A^{\prime}$ and $B^{\prime}$ are glued together, so that $\alpha_{1 A}^{\prime}$ and $\alpha_{1 B}^{\prime}$ become one curve $\alpha_{1}^{\prime}, \lambda_{A}^{\prime}$ and $\lambda_{B}^{\prime}$ become one curve $\lambda^{\prime}$.

We have

$$
\begin{aligned}
\boldsymbol{\alpha}^{\prime} & =\left\{\alpha_{1}^{\prime}, \alpha_{2}^{\prime}, \ldots, \alpha_{2 g+1}^{\prime}, \alpha_{2 g+2}^{\prime}, \ldots, \alpha_{2 g+1+r^{\prime}}^{\prime}\right\} \\
\boldsymbol{\beta}^{\prime} & =\left\{\mu^{\prime}, \beta_{2}^{\prime}, \beta_{3}^{\prime}, \ldots, \beta_{2 g+1+r^{\prime}}^{\prime}\right\}
\end{aligned}
$$


Here $\alpha_{i}^{\prime}$ is the union of two $\operatorname{arcs} \xi_{i}^{\prime} \subset A^{\prime}, \overline{\xi_{i}^{\prime}} \subset B^{\prime}$, for $i=2, \ldots, 2 g+1$. $\alpha_{j}^{\prime}$ lie in $B^{\prime}$, for $j=2 g+2, \ldots, 2 g+1+r^{\prime}$. $\mu^{\prime}$ is the union of two $\operatorname{arcs} \delta^{\prime} \subset A^{\prime}$, $\overline{\delta^{\prime}} \subset B^{\prime} . \quad \mu^{\prime}$ intersects $\alpha_{1}^{\prime}$ transversely in one point, and is disjoint from all other $\alpha^{\prime}$-curves. $\beta_{i}^{\prime}$ 's are disjoint from $\lambda^{\prime}$.

Similarly, we construct a sutured diagram $\left(\Sigma^{\prime \prime}, \boldsymbol{\alpha}^{\prime \prime}, \boldsymbol{\beta}^{\prime \prime}, w^{\prime \prime}, z^{\prime \prime}\right) . \Sigma^{\prime \prime}$ is the union of $A^{\prime \prime}, B^{\prime \prime}$. And the corresponding curves are denoted by $\alpha_{i}^{\prime \prime}, \beta_{i}^{\prime \prime}, \ldots$

Now we glue $A^{\prime}, B^{\prime}, A^{\prime \prime}, B^{\prime \prime}$ together, so that $\alpha_{1 A}^{\prime}$ and $\alpha_{1 B}^{\prime}$ become one curve $\gamma_{1}^{\prime}, \lambda_{B}^{\prime}$ and $\lambda_{A}^{\prime \prime}$ become one curve $\lambda^{\prime}, \alpha_{1 A}^{\prime \prime}$ and $\alpha_{1 B}^{\prime \prime}$ become one curve $\gamma_{1}^{\prime \prime}, \lambda_{B}^{\prime \prime}$ and $\lambda_{A}^{\prime}$ become one curve $\lambda^{\prime \prime} . \overline{\xi_{i}^{\prime}}$ and $\xi_{i}^{\prime \prime}$ are glued together to be a curve $\gamma_{i}^{\prime}$, $\overline{\xi_{i}^{\prime \prime}}$ and $\xi_{i}^{\prime}$ are glued together to be a curve $\gamma_{i}^{\prime \prime}, i=2, \ldots, 2 g+1 . \gamma_{j}^{\prime}=\alpha_{j}^{\prime}$ when $j=2 g+2, \ldots, 2 g+1+r^{\prime}, \gamma_{k}^{\prime \prime}=\alpha_{k}^{\prime \prime}$ when $k=2 g+2, \ldots, 2 g+1+r^{\prime \prime}$. $\beta_{i}^{\prime}$ and $\beta_{i}^{\prime \prime}$ are as before. $\delta^{\prime}, \overline{\delta^{\prime}}, \delta^{\prime \prime}, \overline{\delta^{\prime \prime}}$ are glued together to a closed curve $\mu$. We also pick two basepoints $w, z$ near $\lambda^{\prime} \cap \mu$, but on different sides of $\mu$.

Let

$$
\begin{aligned}
\Sigma & =A^{\prime} \cup B^{\prime} \cup A^{\prime \prime} \cup B^{\prime \prime} \\
\gamma & =\left\{\gamma_{1}^{\prime}, \gamma_{2}^{\prime}, \ldots, \gamma_{2 g+1+r^{\prime}}^{\prime}, \gamma_{2}^{\prime \prime}, \ldots, \gamma_{2 g+1+r^{\prime \prime}}^{\prime \prime}\right\} \\
\boldsymbol{\beta} & =\left\{\mu, \beta_{2}^{\prime}, \ldots, \beta_{2 g+1+r^{\prime}}^{\prime}, \beta_{2}^{\prime \prime}, \ldots, \beta_{2 g+1+r^{\prime \prime}}^{\prime \prime}\right\}
\end{aligned}
$$

Then $(\Sigma, \boldsymbol{\gamma}, \boldsymbol{\beta}, w, z)$ is a Heegaard diagram for $(Y, K)$.

As in the proof of [12, Proposition 3.3], we can wind $\xi_{2}^{\prime}, \ldots, \xi_{2 g+1}^{\prime}$ in $A^{\prime}-\delta^{\prime}$, $\gamma_{2 g+2}^{\prime}, \ldots, \gamma_{2 g+1+r^{\prime}}^{\prime}$ in $B^{\prime}-\bar{\delta}^{\prime}, \xi_{2}^{\prime \prime}, \ldots, \xi_{2 g+1}^{\prime \prime}$ in $A^{\prime \prime}-\delta^{\prime \prime}, \gamma_{2 g+2}^{\prime \prime}, \ldots, \gamma_{2 g+1+r^{\prime \prime}}^{\prime \prime}$ in $B^{\prime \prime}-\overline{\delta^{\prime \prime}}$, so that the diagrams

$$
(\Sigma, \boldsymbol{\gamma}, \boldsymbol{\beta}, w, z),\left(\Sigma^{\prime}, \boldsymbol{\alpha}^{\prime}, \boldsymbol{\beta}^{\prime}, w^{\prime}, z^{\prime}\right),\left(\Sigma^{\prime \prime}, \boldsymbol{\alpha}^{\prime \prime}, \boldsymbol{\beta}^{\prime \prime}, w^{\prime \prime}, z^{\prime \prime}\right)
$$

become admissible, and any nonnegative relative periodic domain in $A^{\prime}$ or $A^{\prime \prime}$ (for these diagrams) is supported away from $\lambda^{\prime \prime}, \lambda^{\prime}$.

Claim If $\mathbf{x}$ is a generator of $\widehat{C F K}(Y, K,-g)$, then $\mathbf{x}$ is supported outside $\operatorname{int}\left(A^{\prime}\right) \cup \operatorname{int}\left(A^{\prime \prime}\right)$.

Let $Y_{0}(K)$ be the manifold obtained from $Y$ by 0 -surgery on $K, \underline{\mathfrak{s}}_{w, z}(\mathbf{y}) \in$ $\operatorname{Spin}^{c}\left(Y_{0}\right)$ be the $\operatorname{Spin}^{c}$ structure associated to an intersection point $\mathbf{y}, \widehat{F^{\prime}}$ be the surface in $Y_{0}$ obtained by capping off the boundary of $F^{\prime}$.

We want to compute $\left\langle c_{1}\left(\underline{\mathfrak{s}}_{w, z}(\mathbf{y})\right),\left[\widehat{F}^{\prime}\right]\right\rangle$.

$\left.(\Sigma, \boldsymbol{\gamma},(\boldsymbol{\beta} \backslash \underline{\{\mu}\}) \cup\left\{\lambda^{\prime \prime}\right\}, w^{\prime \prime}\right)$ is a Heegaard diagram for $Y_{0}$, we wind $\lambda^{\prime \prime}$ once along $\delta^{\prime} \cup \overline{\delta^{\prime}}$ to create two new intersection points with $\gamma_{1}^{\prime}$. The variant of $\lambda^{\prime \prime}$ 
after winding is denoted by $\lambda^{*}$. Let $\mathbf{y}^{*}$ be an intersection point close to $\mathbf{y}$ in this new diagram. A standard computation of $\left\langle c_{1}\left(\mathfrak{s}\left(\mathbf{y}^{*}\right)\right),\left[\widehat{F^{\prime}}\right]\right\rangle$ shows that, an intersection point $\mathbf{x}$ is a generator of $\widehat{C F K}(Y, K,-g)$, if and only if $\mathbf{x}$ is supported outside $\operatorname{int}\left(A^{\prime}\right)$. Now if $\mathbf{x}$ is supported outside $\operatorname{int}\left(A^{\prime}\right)$, then the $\beta_{2}^{\prime}, \ldots, \beta_{2 g+1+r^{\prime}}^{\prime}$ components of $\mathbf{x}$ have to lie in $B^{\prime}$. Hence they are also the $\gamma_{2}^{\prime}, \ldots, \gamma_{2 g+1+r^{\prime}}^{\prime}$ components of $\mathbf{x}$. So $\mathbf{x}$ has no component in $\operatorname{int}\left(A^{\prime \prime}\right)$. This finishes the proof of the claim.

Using the previous claim, one sees that

$$
\widehat{C F K}(Y, K,-g) \cong \widehat{C F K}\left(Y^{\prime}, K^{\prime},-g\right) \otimes \widehat{C F K}\left(Y^{\prime \prime}, K^{\prime \prime},-g\right)
$$

as abelian groups. Suppose $\Phi$ is a holomorphic disk for $\widehat{C F K}(Y, K,-g)$, by the previous claim all the corners of $\Phi$ are supported outside $A^{\prime} \cup A^{\prime \prime}$, so $\Phi \cap\left(A^{\prime} \cup A^{\prime \prime}\right)$ is a nonnegative relative periodic domain in $A^{\prime} \cup A^{\prime \prime}$, our previous conclusion before the claim shows that $\Phi$ is supported away from $\lambda^{\prime \prime}, \lambda^{\prime}$. Moreover, $\Phi$ is supported away from $\gamma_{1}^{\prime} \cap \mu$, since $\Phi$ should avoid $w, z$, which lie on different sides of $\mu$. By the same reason, if $\Phi^{\prime}, \Phi^{\prime \prime}$ are holomorphic disks for $\widehat{C F K}\left(Y^{\prime}, K^{\prime},-g\right)$ and $\widehat{C F K}\left(Y^{\prime \prime}, K^{\prime \prime},-g\right)$, respectively, then they are supported away from $\alpha_{1}^{\prime} \cap \mu^{\prime}$ and $\alpha_{1}^{\prime \prime} \cap \mu^{\prime \prime}$, respectively. Hence $\Phi$ is the disjoint union of two holomorphic disks for $\widehat{C F K}\left(Y^{\prime}, K^{\prime},-g\right)$ and $\widehat{C F K}\left(Y^{\prime \prime}, K^{\prime \prime},-g\right)$, respectively. Now our desired result is obvious.

As a corollary, we have

Corollary 4.4 Let $K \subset Y$ be a null-homologous knot with a genus $g$ Seifert surface $F, Y_{m}$ be the $m$-fold cyclic branched cover of $Y$ over $K$, with respect to $F$, and $K_{m}$ is the image of $K$ in $Y_{m}$. Then

$$
\widehat{H F K}\left(Y_{m}, K_{m},[F], g ; \mathbb{F}\right) \cong \widehat{H F K}(Y, K,[F], g ; \mathbb{F})^{\otimes m}
$$

as linear spaces over any field $\mathbb{F}$.

Knot Floer homology of knots in cyclic branched covers has been studied by Grigsby [7], with emphasis on 2-bridge knots in $S^{3}$.

Theorem 4.1 can be re-stated in the language of $\widehat{H F S}(M, \gamma)$ as follows.

Theorem 4.5 Suppose $(M, \gamma)$ is a balanced sutured manifold, and $S \subset M$ is a horizontal surface. Decompose $(M, \gamma)$ along $S$, we get two balanced sutured manifolds $\left(M_{1}, \gamma_{1}\right),\left(M_{2}, \gamma_{2}\right)$. Then

$$
\widehat{H F S}(M, \gamma) \cong \widehat{H F S}\left(M_{1}, \gamma_{1}\right) \otimes \widehat{H F S}\left(M_{2}, \gamma_{2}\right)
$$

as linear spaces over any field $\mathbb{F}$. 


\section{Product decomposition}

In this section, we will study sutured manifold decomposition along product annuli. We are not able to obtain a formula for non-separating product annuli, but the formula for separating product annuli is already enough for many applications.

Theorem 5.1 Suppose $(M, \gamma)$ is a balanced sutured manifold, $R_{ \pm}(\gamma)$ are connected. $\mathcal{A} \subset M$ is a separating product annulus, and $\mathcal{A}$ separates $M$ into two balanced sutured manifolds $\left(M_{1}, \gamma_{1}\right),\left(M_{2}, \gamma_{2}\right)$.

Then we have

$$
\widehat{H F S}(M, \gamma) \cong \widehat{H F S}\left(M_{1}, \gamma_{1}\right) \otimes \widehat{H F S}\left(M_{2}, \gamma_{2}\right)
$$

as vector spaces over any field $\mathbb{F}$.

In the first two subsections, we will consider the case that $\gamma$ has only one component, which lies in $M_{2}$, and $M_{1}=R_{1} \times[0,1]$, where $R_{1}$ is a compact genus -1 surface with one boundary component.

\subsection{A Heegaard diagram related to $(M, \gamma)$}

Construction 5.2 Let $\psi: R_{+}(\gamma) \rightarrow R_{-}(\gamma)$ be a homeomorphism, such that $\psi\left(R_{+}\left(\gamma_{i}\right)\right)=R_{-}\left(\gamma_{i}\right), i=1,2$, and $\psi \mid R_{+}\left(\gamma_{1}\right)$ maps $x \times 1$ to $x \times 0$ for any $x \in R_{1}$. If we glue $R_{+}(\gamma)$ to $R_{-}(\gamma)$ by $\psi$, then we get a manifold with boundary consisting of a torus. This manifold can be viewed as the complement of a knot $K$ in a manifold $Y$. We will construct a Heegaard diagram for the pair $(Y, K)$. The construction is similar to Construction 2.11.

Step 0. A relative Morse function

Consider a self-indexed relative Morse function $u$ on $\left(M_{2}, \gamma_{2}\right)$. Let $\widetilde{F}_{2}=$ $u^{-1}\left(\frac{3}{2}\right) . \quad \widetilde{F}_{2}$ has two boundary components. We denote the one that lies in the separating annulus $\mathcal{A}$ by $\widetilde{a}$. The other boundary component is denoted by $\widetilde{\lambda}$. Similarly, the boundary components of $R_{ \pm}\left(\gamma_{2}\right)$ are denoted by $a_{ \pm}, \lambda_{ \pm}$.

Suppose the genus of $R_{+}\left(\gamma_{2}\right)$ is $g-1$, and $u$ has $r$ index-1 critical points, then the genus of $\widetilde{F}_{2}$ is $g+r-1$. The gradient $-\nabla u$ generates a flow $\phi_{t}$ on $M_{2}$.

Step 1. Construct curves for $\left(M_{2}, \gamma_{2}\right)$

Choose

$$
\widetilde{D}, \widetilde{\delta} \subset \widetilde{F}_{2}, \quad D_{ \pm}, \delta_{ \pm} \subset R_{ \pm}\left(\gamma_{2}\right)
$$




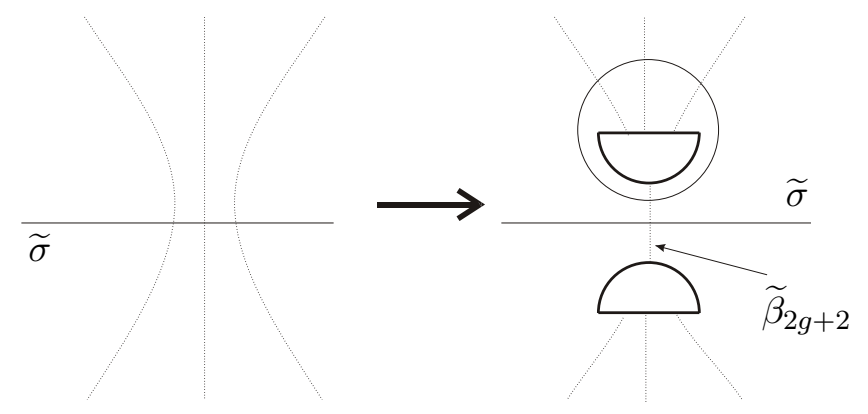

Figure 1: A stabilization will eliminate the extra intersection points of $\widetilde{\sigma}$ with $\widetilde{\tau}$ and $\widetilde{\beta}$-curves. On the right hand side the two semicircular holes are glued together.

as in Construction 2.11, Let $B_{ \pm}=R_{ \pm}\left(\gamma_{2}\right)-\operatorname{int}\left(D_{ \pm}\right), \widetilde{B}=\widetilde{F}_{2}-\operatorname{int}(\widetilde{D})$.

On $\widetilde{F}_{2}$, there are simple closed curves $\widetilde{\alpha}_{2 g+2}, \ldots, \widetilde{\alpha}_{2 g+1+r}, \widetilde{\beta}_{2 g+2}, \ldots, \widetilde{\beta}_{2 g+1+r}$, which correspond to the critical points of $u$.

The $\widetilde{\alpha}$-curves do not separate $\widetilde{F}_{2}$, so there is an arc $\widetilde{\sigma} \subset \widetilde{B}$ connecting $\widetilde{\lambda}$ to $\widetilde{a}$, and $\widetilde{\sigma}$ is disjoint from $\widetilde{\delta}$ and $\widetilde{\alpha}$-curves. Similarly, there is an arc $\widetilde{\tau} \subset \widetilde{F}_{2}$ connecting $\partial \widetilde{D}$ to $\widetilde{a}$, and $\widetilde{\tau}$ is disjoint from $\widetilde{\delta}$ and $\widetilde{\beta}$-curves. Moreover, by stabilization as shown in Figure 1, we can assume $\widetilde{\sigma} \cap \widetilde{\tau}=\emptyset$, and $\widetilde{\sigma}$ intersects exactly one $\widetilde{\beta}$-curve transversely once. Suppose this curve is $\widetilde{\beta}_{2 g+2}$. Let $\sigma_{-} \subset$ $B_{-}$be the image of $\widetilde{\sigma}$ under the flow $\phi_{t}$, and $\sigma_{+}=\psi^{-1}\left(\sigma_{-}\right)$. Let $\tau_{+} \subset B_{+}$be the image of $\widetilde{\tau}$ under the flow $\phi_{-t}$.

Choose $2 g-2$ disjoint arcs $\xi_{4}^{-}, \ldots, \xi_{2 g+1}^{-} \subset B_{-}$, such that their endpoints lie on $\lambda_{-}$, and they are linearly independent in $\mathrm{H}_{1}\left(B_{-}, \partial B_{-}\right)$. We also suppose they are disjoint from $\delta_{-}, \sigma_{-}$and the bad points. Let $\xi_{i}^{+}=\psi^{-1}\left(\xi_{i}^{-}\right)$. We also flow back $\xi_{4}^{-}, \ldots, \xi_{2 g+1}^{-}$by $\phi_{-t}$ to $\widetilde{F}_{2}$, the images are denoted by $\widetilde{\xi}_{4}, \ldots, \widetilde{\xi}_{2 g+1}$.

Choose $2 g-2$ disjoint arcs $\eta_{4}^{+}, \ldots, \eta_{2 g+1}^{+} \subset B_{+}$, such that their endpoints lie on $\partial D_{+}$, and they are linearly independent in $\mathrm{H}_{1}\left(B_{+}, \partial B_{+}\right)$. We also suppose they are disjoint from $\delta_{+}, \tau_{+}$and the bad points. Flow them by $\phi_{t}$ to $\widetilde{F}$, the images are denoted by $\widetilde{\eta}_{4}, \ldots, \widetilde{\eta}_{2 g+1}$.

We can slide $\widetilde{\eta}$-curves over $\widetilde{\beta}_{2 g+2}$ to eliminate the possible intersection points between $\widetilde{\eta}$-curves and $\widetilde{\sigma}$.

By stabilization, we can assume $\widetilde{\tau}$ does not intersect $\widetilde{\xi}$-curves, and it intersects exactly one $\widetilde{\alpha}$-curve transversely once. This curve is denoted by $\widetilde{\alpha}_{2 g+2}$. 


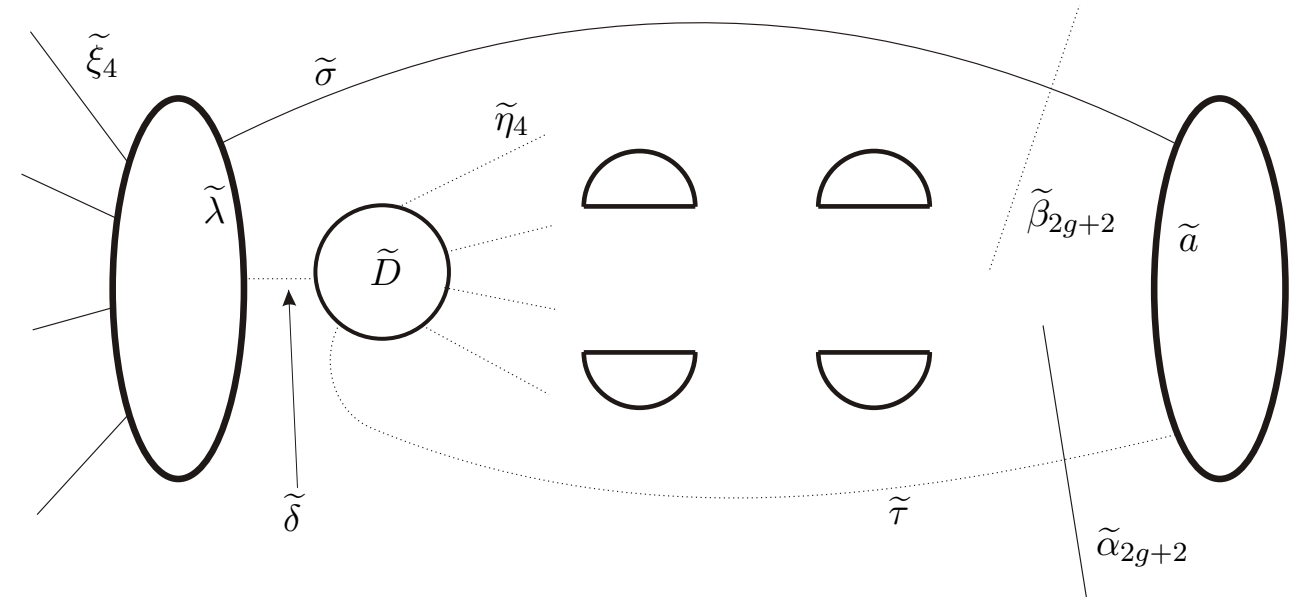

Figure 2: A schematic picture of $\widetilde{F}_{2}$. The pairs of semicircular holes are glued together. 
Step 2. Find geometric duals of $\widetilde{\alpha}$-curves

As in Lemma 2.12, we can prove that

$$
E=\widetilde{B}-\cup_{i=4}^{2 g+1} \widetilde{\xi}_{i}-\cup_{j=2 g+2}^{2 g+1+r} \widetilde{\alpha}_{j}-\widetilde{\delta}-\widetilde{\sigma}
$$

is connected. Then there are simple closed curves

$$
\theta_{2 g+2}, \ldots, \theta_{2 g+1+r} \subset \widetilde{B}-\cup \widetilde{\xi}_{i}-\widetilde{\sigma}-\widetilde{\delta},
$$

which are geometrically dual to $\widetilde{\alpha}_{2 g+2}, \ldots, \widetilde{\alpha}_{2 g+1+r}$.

We can slide $\widetilde{\theta}_{2 g+3}, \ldots, \widetilde{\theta}_{2 g+1+r}$ over $\widetilde{\alpha}_{2 g+2}$ to eliminate the possible intersection points between $\widetilde{\theta}_{2 g+3}, \ldots, \widetilde{\theta}_{2 g+1+r}$ and $\widetilde{\tau}$.

Step 3. Construct curves for $\left(M_{1}, \gamma_{1}\right)$

As in Figure 3, we choose 4 properly embedded $\operatorname{arcs} \xi_{2}, \xi_{3}, \eta_{2}, \eta_{3}$ on $R_{1}$. $\xi_{2}$ intersects $\eta_{2}$ at one point, and $\xi_{3}$ intersects $\eta_{3}$ at one point. There are no more intersection points between these arcs.

Let

$$
\begin{aligned}
\widetilde{A} & =\widetilde{B} \cup_{\widetilde{a}=\left(\partial R_{1}\right) \times \frac{1}{2}}\left(R_{1} \times \frac{1}{2}\right), \\
A_{+} & =B_{+} \cup_{a_{+}=\left(\partial R_{1}\right) \times 1}\left(R_{1} \times 1\right) .
\end{aligned}
$$

Take 4 parallel copies of $\widetilde{\sigma}$, glue them to $\xi_{2} \times \frac{1}{2}, \xi_{3} \times \frac{1}{2}$, we get two $\operatorname{arcs} \widetilde{\xi}_{2}, \widetilde{\xi}_{3} \subset \widetilde{A}$. Glue 4 parallel copies of $\widetilde{\tau}$ with $\eta_{2} \times \frac{1}{2}, \eta_{3} \times \frac{1}{2}$, we get two $\operatorname{arcs} \widetilde{\eta}_{2}, \widetilde{\eta}_{3} \subset \widetilde{A}$. Similarly, we can construct $\xi_{2}^{+}, \xi_{3}^{+}, \eta_{2}^{+}, \eta_{3}^{+} \subset A_{+}$.

Step 4. Construct a Heegaard diagram

Let

$$
\Sigma=A_{+} \cup \widetilde{A} \cup\{2 \text { annuli }\},
$$

where one annulus is $\phi_{[0,3 / 2]}\left(\partial D_{+}\right)$, the other is $\phi_{[0,3 / 2]}(\widetilde{\lambda})$. Construct $\alpha_{i}, \beta_{i}$, $(i=2, \ldots, 2 g+1) \alpha_{1}, \lambda, \mu$ as in Step 2 of Construction 2.11.

Let

$$
\begin{aligned}
\boldsymbol{\alpha} & =\left\{\alpha_{2}, \ldots, \alpha_{2 g+1}\right\} \cup\left\{\widetilde{\alpha}_{2 g+2}, \ldots, \widetilde{\alpha}_{2 g+1+r}\right\} \cup\left\{\alpha_{1}\right\}, \\
\boldsymbol{\beta} & =\left\{\beta_{2}, \ldots, \beta_{2 g+1}\right\} \cup\left\{\widetilde{\beta}_{2 g+2}, \ldots, \widetilde{\beta}_{2 g+1+r}\right\} \cup\{\mu\} .
\end{aligned}
$$

Pick two base points $w, z$ near $\lambda \cap \mu$, but on different sides of $\mu$. As in Construction 2.11.

$$
(\Sigma, \boldsymbol{\alpha}, \boldsymbol{\beta}, w, z)
$$

is a Heegaard diagram for $(Y, K)$. 


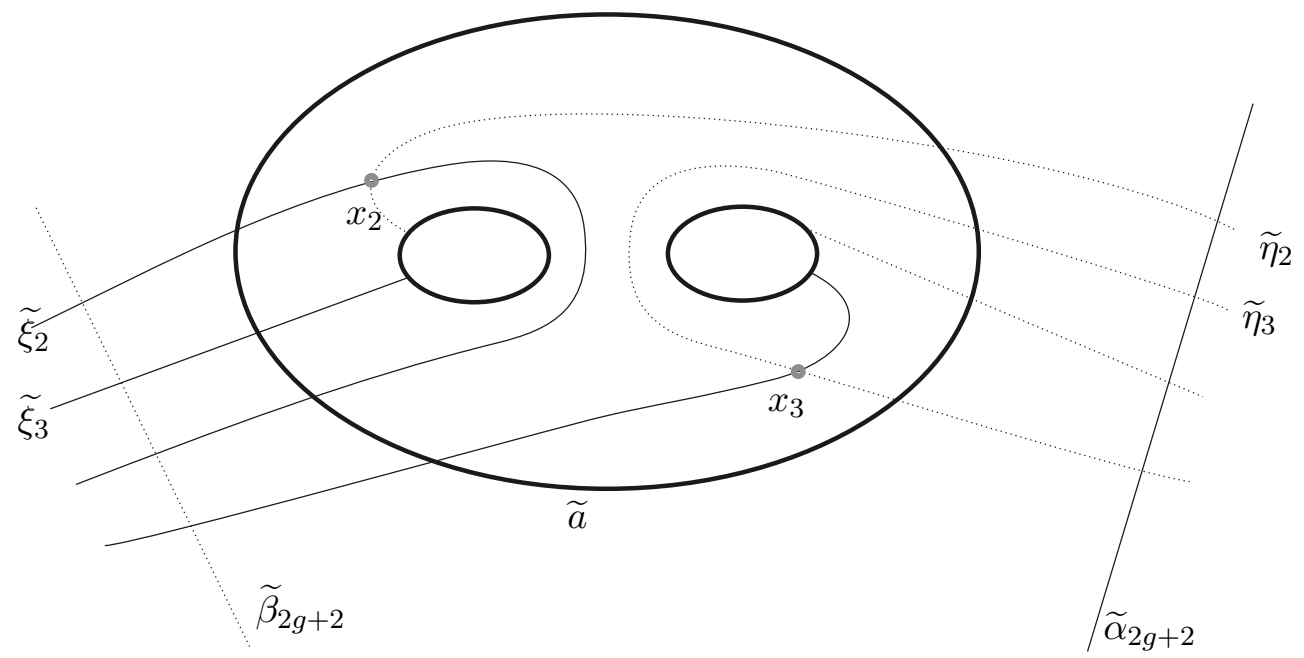

Figure 3: Local picture of $(\Sigma, \boldsymbol{\alpha}, \boldsymbol{\beta}, w, z)$ near $R_{1} \times \frac{1}{2}$. The surface $R_{1}$ is obtained by gluing the boundaries of two holes in the disk by a reflection.

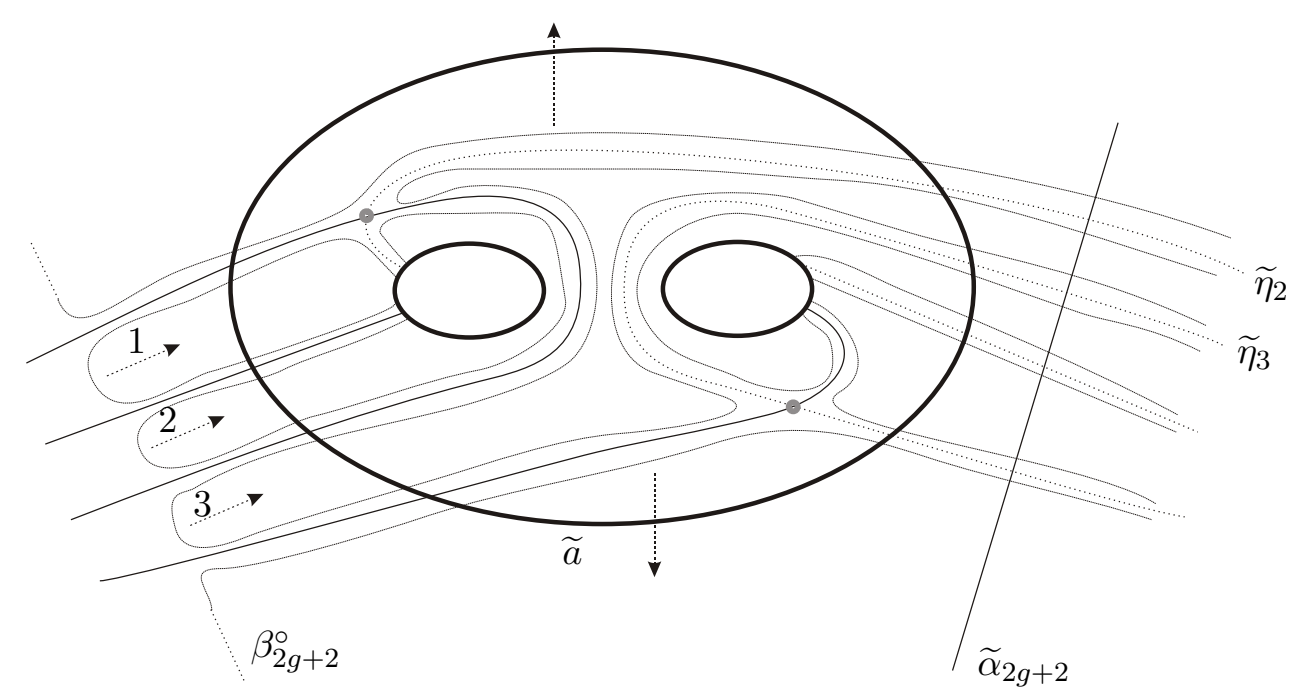

Figure 4: Local picture of $\left(\Sigma, \boldsymbol{\alpha}, \boldsymbol{\beta}^{\circ}, w, z\right)$ near $R_{1} \times \frac{1}{2}$. The arrows indicate the directions of a further isotopy. 


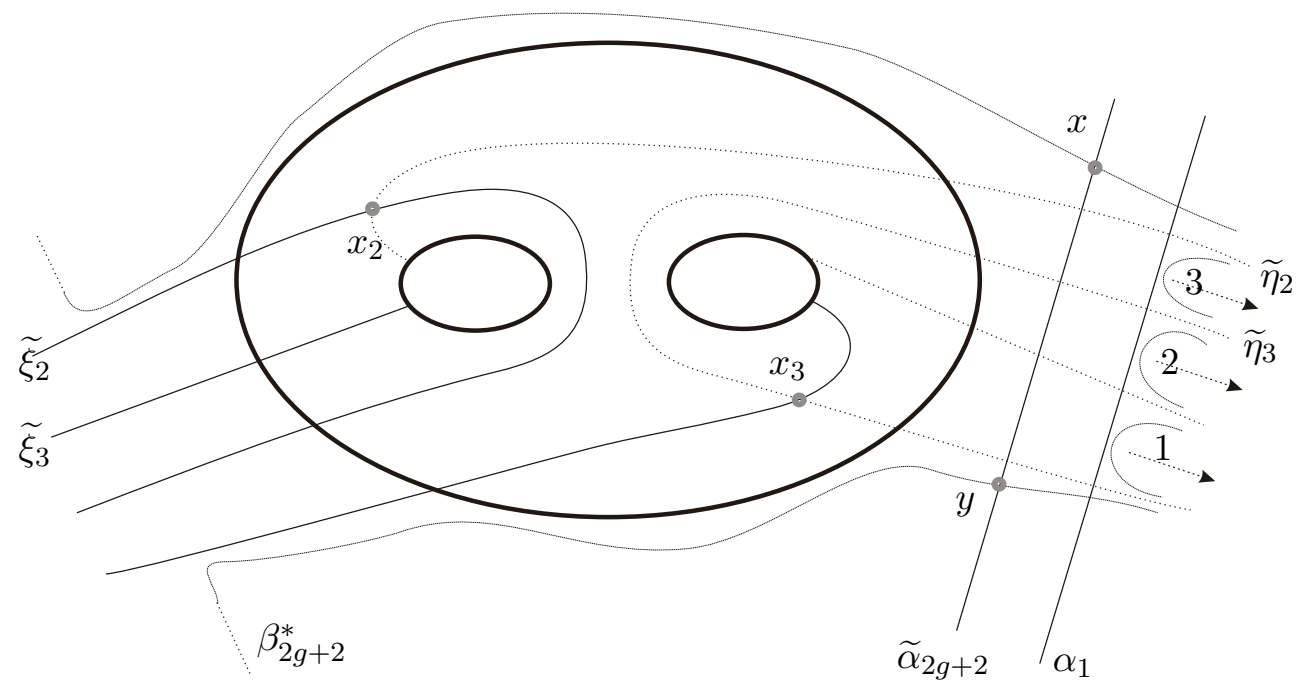

Figure 5: Local picture of $\left(\Sigma, \boldsymbol{\alpha}, \boldsymbol{\beta}^{*}, w, z\right)$.

It is easy to check that the Heegaard diagram constructed above is a sutured Heegaard diagram. In order to prove our desired result, we still need to change the diagram by handleslides.

Construction 5.3 In the Heegaard diagram $(\Sigma, \boldsymbol{\alpha}, \boldsymbol{\beta}, w, z)$ constructed in Construction 5.2, the curve $\widetilde{\beta}_{2 g+2}$ has 4 intersection points with $\widetilde{\xi}_{2}$ and $\widetilde{\xi}_{3}$. Since $\widetilde{\xi}_{2}$ intersects $\beta_{2}$ exactly once, we can slide $\widetilde{\beta}_{2 g+2}$ over $\beta_{2}$ twice, to eliminate the intersection points between $\widetilde{\beta}_{2 g+2}$ and $\widetilde{\xi}_{2}$. Note that in the two handleslides, the orientations of $\beta_{2}$ are different, so the new curve is homologous to $\widetilde{\beta}_{2 g+2}$. Similarly, we can slide $\widetilde{\beta}_{2 g+2}$ over $\beta_{3}$ twice, to eliminate the intersection points between $\widetilde{\beta}_{2 g+2}$ and $\widetilde{\xi}_{3}$. The local picture is shown in Figure 4 . The new $\widetilde{\beta}$-curve is denoted by $\beta_{2 g+2}^{\circ}$.

Moreover, we can isotope $\beta_{2 g+2}^{\circ}$ to eliminate its intersection points with $\widetilde{\alpha}_{2 g+2}$ and $\alpha_{1}$, then isotope it so that it lies outside $R_{1} \times \frac{1}{2}$, as shown in Figure 4 and Figure 5. The new $\widetilde{\beta}$-curve is denoted by $\beta_{2 g+2}^{*} \cdot \beta_{2 g+2}^{*}$ is homologous to $\widetilde{\beta}_{2 g+2}$ in $\mathrm{H}_{1}(\Sigma)$, thus $\beta_{2 g+2}^{*} \cap A_{+}$is null-homologous in $\mathrm{H}_{1}\left(A_{+}, \partial A_{+}\right)$.

The new Heegaard diagram after handlesliding is denoted by $\left(\Sigma, \boldsymbol{\alpha}, \boldsymbol{\beta}^{*}, w, z\right)$. This diagram is still a sutured Heegaard diagram. 
Lemma 5.4 After winding transverse to $\widetilde{\alpha}$-curves in $\widetilde{B}$, and transverse to $\xi^{+}$ curves in $A_{+}$, we can get a weakly admissible Heegaard diagram for $(Y, K)$. Moreover, in this diagram, any holomorphic disk for $\widehat{C F K}(Y, K,-g)$ is supported in $\widetilde{B}$.

Proof In Step 2 of Construction 5.2, we find circles $\theta_{2 g+2}, \ldots, \theta_{2 g+1+r}$, which are geometrically dual to $\widetilde{\alpha}_{2 g+2}, \ldots, \widetilde{\alpha}_{2 g+1+r}$. Moreover, $\theta_{2 g+3}, \ldots, \theta_{2 g+1+r}$ are disjoint with $\widetilde{\xi}$-curves, $\widetilde{\delta}$, $\widetilde{\sigma}$ and $\widetilde{\tau}$. As in the proof of [12, Proposition 3.3], we can wind $\widetilde{\alpha}_{2 g+3}, \ldots, \widetilde{\alpha}_{2 g+1+r}$ along $\theta_{2 g+3}, \ldots, \theta_{2 g+1+r}$, and wind $\xi^{+}$-curves in $A_{+}$, to get a new Heegaard diagram. In this diagram, if $\mathcal{P}$ is a nonnegative periodic domain, then $\partial \mathcal{P}$ does not contain $\widetilde{\alpha}_{2 g+3}, \ldots, \widetilde{\alpha}_{2 g+1+r}$ and $\xi^{+}$-curves. Obviously, $\partial P$ does not contain $\mu$. Note that $\alpha_{1}$ is the only attaching curve in the Heegaard diagram which intersects $\mu$, and $\partial P \cdot \mu=0 \neq \alpha_{1} \cdot \mu$, so $\partial P$ does not contain $\alpha_{1}$.

$\partial\left(\mathcal{P} \cap A_{+}\right)-\partial A_{+}$is a linear combination of $\beta_{2 g+2}^{*} \cap A_{+}$and $\eta_{i}^{+}$'s. Note that $\eta_{2}^{+}, \ldots, \eta_{2 g+1}^{+}$are linearly independent in $\mathrm{H}_{1}\left(A_{+}, \partial A_{+}\right)$, and $\beta_{2 g+2}^{*} \cap A_{+}$is nullhomologous in $\mathrm{H}_{1}\left(A_{+}, \partial A_{+}\right)$, so $\partial\left(\mathcal{P} \cap A_{+}\right)-\partial A_{+}$is a multiple of $\beta_{2 g+2}^{*} \cap A_{+}$.

Hence $\partial \mathcal{P}$ is a linear combination of $\widetilde{\alpha}_{2 g+2}$ and $\widetilde{\beta}$-curves. Here $\beta_{2 g+2}^{*}$ is also viewed as a $\widetilde{\beta}$-curve, since it is homologous to $\widetilde{\beta}_{2 g+2}$. In Step 1 of Construction [5.2, $\widetilde{\alpha}_{2 g+2}$ is obtained by stabilization. Thus there is a $\widetilde{\beta}$-curve, which intersects $\widetilde{\alpha}_{2 g+2}$ exactly once. $\widetilde{\beta}$-curves are mutually disjoint, so $\widetilde{\alpha}_{2 g+2}$ is not homologous to the linear combination of $\widetilde{\beta}$-curves. This shows that the new diagram is weakly admissible. This diagram is still denoted by $\left(\Sigma, \boldsymbol{\alpha}, \boldsymbol{\beta}^{*}, w, z\right)$.

The generators of $\widehat{C F K}(Y, K,-g)$ are supported outside $\operatorname{int}\left(A_{+}\right)$.

$\alpha_{2}$ and $\alpha_{3}$ intersect only two $\beta$-curves outside $A_{+}$. Therefore the two intersection points $x_{2}=\widetilde{\xi}_{2} \cap \widetilde{\eta}_{2}$ and $x_{3}=\widetilde{\xi}_{3} \cap \widetilde{\eta}_{3}$ must be chosen.

Suppose $\Phi$ is a holomorphic disk for $\widehat{C F K}(Y, K,-g)$. $\Phi \cap A_{+}$is a relative periodic domain [12, Definition 3.1] in $A_{+}$. As before, $\Phi$ does not contain any $\xi_{+}-$curve after winding, so $\Phi$ is supported away from $\lambda$. Now $\partial\left(\Phi \cap A_{+}\right)-\partial A_{+}$ is a linear combination of $\beta_{2 g+2}^{*} \cap A_{+}$and $\eta_{i}^{+}$'s. As in the second paragraph of this proof, we have that

$$
\partial\left(\Phi \cap A_{+}\right)-\partial A_{+}=m\left(\beta_{2 g+2}^{*} \cap A_{+}\right) .
$$

$\Phi$ is supported away from $\mu, \mu$ intersects $\alpha_{1}$ at exactly one point, so the contribution of $\alpha_{1}$ to $\partial \Phi$ is 0 . $\beta_{2 g+2}^{*} \cap A_{+}$separates $\lambda$ from $\eta_{2}^{+} \cap \alpha_{1}$. The local multiplicities of $\Phi$ in a small neighborhood of $x_{2}, x_{3}$ are 0 , and $\Phi$ does 


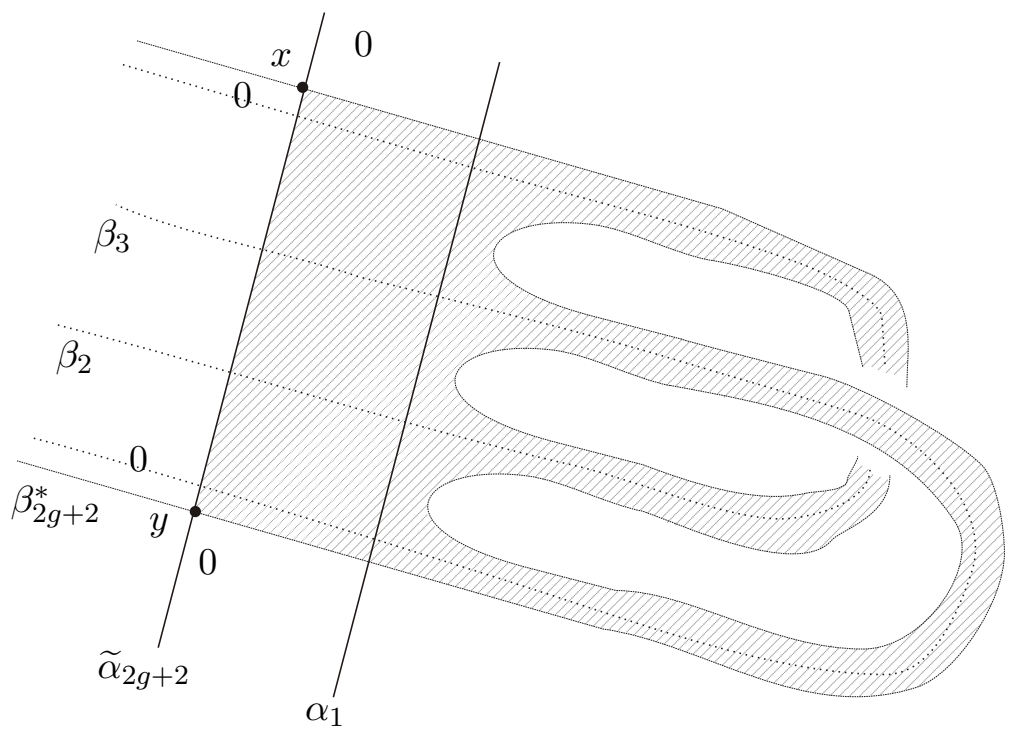

Figure 6: A component of the possible holomorphic disk. The two bands lie in $R_{1} \times 1$.

not intersect $\lambda$. As in Figure 6, we conclude that the local multiplicities of $\Phi$ in two corners at $x$ are 0 , and the local multiplicities of $\Phi$ in two corners at $y$ are 0 .

If $m>0$, then $\Phi$ (which is possibly reducible) has a component $\Phi_{0}$, which has two vertices $x, y$ and two edges lying in $\widetilde{\alpha}_{2 g+2}$ and $\beta_{2 g+2}^{*}$. See Figure 6 for the picture of $\Phi_{0}$. The genus of $\Phi_{0}$ is 1 , and its Maslov index is -1 . So $\Phi$ has no contribution to the boundary map in $\widehat{C F K}(Y, K,-g)$.

Hence if $\Phi$ is a holomorphic disk for $\widehat{C F K}(Y, K,-g)$, then $m=0$, so $\Phi$ is supported in $\widetilde{B}$.

\subsection{A Heegaard diagram related to $\left(M_{2}, \gamma_{2}\right)$}

We also need to construct a Heegaard diagram to compute $\widehat{H F S}\left(M_{2}, \gamma_{2}\right)$. As in Subsection 2.2, we can add a product 1-handle to $M_{2}$ with feet at different components of $\gamma_{2}$ to get a new sutured manifold $\left(M_{3}, \gamma_{3}\right)$. Then we glue $R_{+}\left(\gamma_{3}\right)$ with $R_{-}\left(\gamma_{3}\right)$ to get the complement of a knot $K_{3}$ in a manifold $Y_{3}$. Then

$$
\widehat{H F S}\left(M_{2}, \gamma_{2}\right)=\widehat{H F K}\left(Y_{3}, K_{3}, g\right) \text {. }
$$


Our next task is to construct a Heegaard diagram for $\left(Y_{3}, K_{3}\right)$.

Construction 5.5 Notations as in Construction [5.2, As in Figure 7, let $\widetilde{C}$ be the surface obtained by gluing a rectangle $I \times[0,1]$ to $\widetilde{B}$, such that

$$
(I \times[0,1]) \cap \widetilde{B}=I \times\{0,1\},(I \times 0) \subset \widetilde{\lambda},(I \times 1) \subset \widetilde{a} .
$$

Moreover, $\widetilde{\delta} \cap \widetilde{\lambda}$ is not contained in $I \times 0$, but it is close to $I \times 0$. $\widetilde{\tau} \cap \widetilde{a} \in \operatorname{int}(I \times 1)$, and $\widetilde{\sigma} \cap \widetilde{a} \notin I \times 1$. Let $\widetilde{\zeta}_{2}=I \times \frac{1}{2}, \widetilde{\zeta}_{3}=\widetilde{\sigma}$. Let $\widetilde{\omega}_{2}$ be the union of $\widetilde{\tau}, p \times[0,1]$ $(p \in \operatorname{int}(I))$ and a parallel copy of $\widetilde{\delta}$. Let $T$ be the neighborhood of $\widetilde{\tau} \cup \widetilde{a}$ in $\widetilde{B}$. $\widetilde{\omega}_{3}=T \cap(\widetilde{B}-\operatorname{int}(T))$.

Similarly, construct a surface $C_{+}$, and curves $\zeta_{2}^{+}, \zeta_{3}^{+}, \omega_{2}^{+}, \omega_{3}^{+}$on it.

As in Step 4 of Construction 5.2 , for $i=2,3$, let

$$
\begin{aligned}
& \alpha_{i}^{\prime}=\zeta_{i}^{+} \cup \widetilde{\zeta}_{i} \cup\{2 \operatorname{arcs}\}, \\
& \beta_{i}^{\prime}=\omega_{i}^{+} \cup \widetilde{\omega}_{i} \cup\{2 \operatorname{arcs}\} .
\end{aligned}
$$

Let

$$
\begin{aligned}
\Sigma^{\prime} & =C_{+} \cup \widetilde{C} \cup\{2 \text { annuli }\}, \\
\boldsymbol{\alpha}^{\prime} & =\left\{\alpha_{2}^{\prime}, \alpha_{3}^{\prime}, \alpha_{4}, \ldots, \alpha_{2 g+1}\right\} \cup\left\{\widetilde{\alpha}_{2 g+2}, \ldots, \widetilde{\alpha}_{2 g+1+r}\right\} \cup\left\{\alpha_{1}\right\}, \\
\boldsymbol{\beta}^{\prime} & =\left\{\beta_{2}^{\prime}, \beta_{3}^{\prime}, \beta_{4}, \ldots, \beta_{2 g+1}\right\} \cup\left\{\widetilde{\beta}_{2 g+2}, \ldots, \widetilde{\beta}_{2 g+1+r}\right\} \cup\{\mu\} .
\end{aligned}
$$

Then

$$
\left(\Sigma^{\prime}, \boldsymbol{\alpha}^{\prime}, \boldsymbol{\beta}^{\prime}, w, z\right)
$$

is a Heegaard diagram for $\left(Y_{3}, K_{3}\right)$.

Construction 5.6 In the diagram $\left(\Sigma^{\prime}, \boldsymbol{\alpha}^{\prime}, \boldsymbol{\beta}^{\prime}, w, z\right)$, we can slide $\widetilde{\beta}_{2 g+2}$ over $\beta_{3}^{\prime}$ once, to get a new curve $\beta_{2 g+2}^{\prime \prime}$. The new diagram is denoted by $\left(\Sigma^{\prime}, \boldsymbol{\alpha}^{\prime}, \boldsymbol{\beta}^{\prime \prime}, w, z\right)$. See Figure 8 for the local picture.

Lemma 5.7 After winding transverse to $\widetilde{\alpha}$-curves in $\widetilde{B}$, and transverse to $\xi^{+}-$ curves in $B_{+}$, we can get a weakly admissible Heegaard diagram for $\left(Y_{3}, K_{3}\right)$. In this diagram, any holomorphic disk for $\widehat{C F K}\left(Y_{3}, K_{3},-g\right)$ is supported in $\widetilde{B}$. 


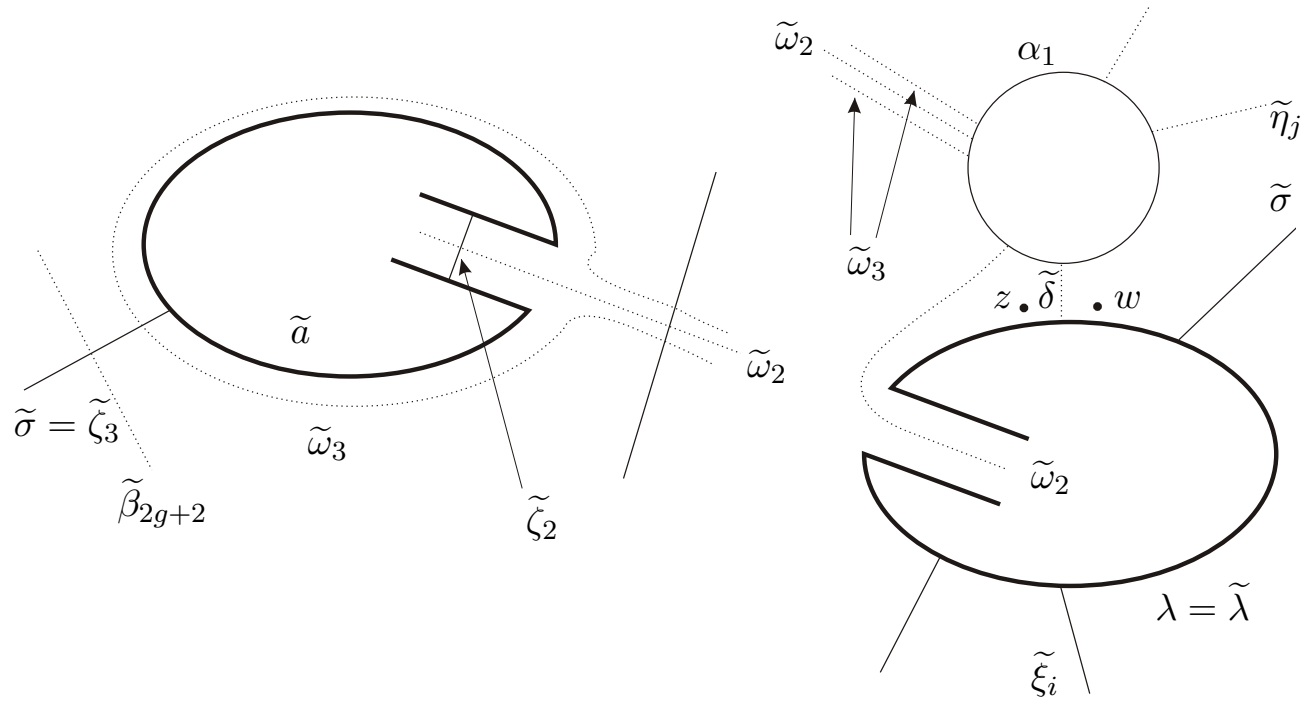

Figure 7: Local picture of $\left(\Sigma^{\prime}, \boldsymbol{\alpha}^{\prime}, \boldsymbol{\beta}^{\prime}, w, z\right)$, near $\widetilde{a}$ and near $\widetilde{\lambda}$. There is a band connecting $\widetilde{a}$ to $\tilde{\lambda}$.

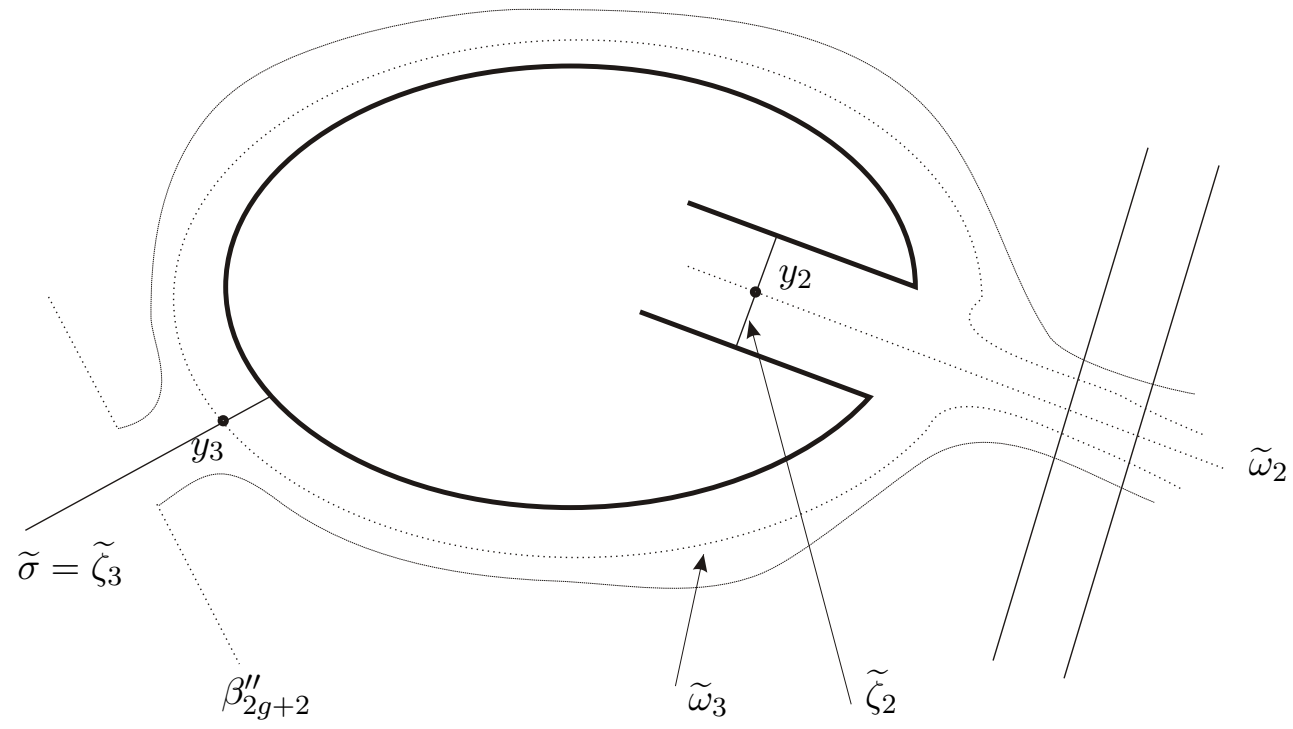

Figure 8: Local picture of $\left(\Sigma^{\prime}, \boldsymbol{\alpha}^{\prime}, \boldsymbol{\beta}^{\prime \prime}, w, z\right)$ near $\widetilde{a}$. 
Proof The proof of this lemma is similar to the proof of Lemma 5.4, and the argument here is even simpler. Again, we can wind some curves, such that the diagram becomes weakly admissible, and the holomorphic disks for $\widehat{C F K}\left(Y_{3}, K_{3},-g\right)$ are supported away from $\lambda^{\prime}$. Here $\lambda^{\prime} \subset \partial \widetilde{C}$ is the connected sum of $\lambda$ and $\widetilde{a}$. Suppose $\Phi^{\prime}$ is a holomorphic disk for $\widehat{C F K}\left(Y_{3}, K_{3},-g\right)$, then $\Phi^{\prime} \cap C_{+}$is a relative periodic domain in $C_{+}$. Since $\Phi^{\prime}$ is disjoint with $\lambda^{\prime}, \partial \Phi^{\prime} \cap C_{+}$does not contain $\xi^{+}$-curves, and the local multiplicities of $\Phi^{\prime}$ near $y_{2}$ and $y_{3}$ are zero. So $\partial\left(\Phi^{\prime} \cap C_{+}\right)-\partial C_{+}$is a linear combination of $\eta_{4}^{+}, \ldots, \eta_{2 g+1}^{+}, \beta_{2 g+2}^{\prime \prime} \cap C_{+}$. These arcs are linearly independent in $\mathrm{H}_{1}\left(C_{+}, \partial C_{+}\right)$, so $\Phi^{\prime}$ is supported in $\widetilde{B}$.

\subsection{Proof of the product decomposition formula}

Proof of Theorem 5.1 The surfaces

$$
F_{ \pm}=R_{ \pm}\left(\gamma_{1}\right) \cup \mathcal{A} \cup R_{\mp}\left(\gamma_{2}\right)
$$

are homologous to $R_{+}(\gamma) . F_{ \pm}$can be isotoped to horizontal surfaces in $(M, \gamma)$. $F_{+}$decomposes $(M, \gamma)$ into two sutured manifolds $\left(M_{1}^{\prime}, \gamma_{1}^{\prime}\right),\left(M_{2}^{\prime}, \gamma_{2}^{\prime}\right)$. Here $\left(M_{1}^{\prime}, \gamma_{1}^{\prime}\right)$ is the sutured manifold obtained by gluing $R_{-}\left(\gamma_{2}\right) \times I$ to $\left(M_{1}, \gamma_{1}\right)$ along $\mathcal{A}$, and $\left(M_{2}^{\prime}, \gamma_{2}^{\prime}\right)$ is the sutured manifold obtained by gluing $R_{+}\left(\gamma_{1}\right) \times I$ to $\left(M_{2}, \gamma_{2}\right)$ along $\mathcal{A}$. Now one can apply Theorem 4.5 to conclude that

$$
\widehat{H F S}(M, \gamma) \cong \widehat{H F S}\left(M_{1}^{\prime}, \gamma_{1}^{\prime}\right) \otimes \widehat{H F S}\left(M_{2}^{\prime}, \gamma_{2}^{\prime}\right) \text {. }
$$

We only need to show that $\widehat{H F S}\left(M_{i}^{\prime}, \gamma_{i}^{\prime}\right) \cong \widehat{H F S}\left(M_{i}, \gamma_{i}\right)$ for $i=1,2$. Hence we can reduce our theorem to the case that one of the two sutured submanifolds $M_{1}, M_{2}$ is a product.

From now on, we assume $M_{1}$ is a product.

According to Definition 2.2, $\gamma \neq \emptyset$. If $M_{1} \cap \gamma \neq \emptyset$, then one can decompose $M$ along product disks to get $M_{2}$. Now we apply Proposition 2.9 (2) to conclude that $\widehat{H F S}(M, \gamma) \cong \widehat{H F S}\left(M_{2}, \gamma_{2}\right)$.

Now we consider the case that $M_{1} \cap \gamma=\emptyset$. By adding product 1-handles with feet at $\gamma$, we can get a sutured manifold with connected suture.

$R_{+}\left(\gamma_{1}\right)$ contains a subsurface $G$ which is a once-punctured torus. $\partial G \times I$ splits $M$ into two sutured manifolds $G \times I$ and $\left(M^{*}, \gamma^{*}\right)$. One can then decompose $M^{*}$ along product disks to get $\left(M_{2}, \gamma_{2}\right)$. Hence we only need to prove the decomposition formula for the case of splitting along $\partial G \times I$. From now on, we focus on this case, namely, the case that the genus of $R_{+}\left(\gamma_{1}\right)$ is 1 . 
We apply the constructions in the previous two subsections to get Heegaard diagrams for $(Y, K)$ and $\left(Y_{3}, K_{3}\right)$. See Figure 5 and Figure 8 for the local pictures. For generators of $\widehat{C F K}(Y, K,-g)$, the two intersection points $x_{2}, x_{3}$ in Figure 5 must be chosen; for generators of $\widehat{C F K}\left(Y_{3}, K_{3},-g\right)$, the two intersection points $y_{2}, y_{3}$ in Figure 8 must be chosen. Thus the generators of $\widehat{C F K}(Y, K,-g)$ and $\widehat{C F K}\left(Y_{3}, K_{3},-g\right)$ are in one-to-one correspondence. By Lemma 5.4 and Lemma 5.7, the holomorphic disks for these two chain complexes are also the same. Hence

$$
\widehat{H F K}(Y, K,-g)=\widehat{H F K}\left(Y_{3}, K_{3},-g\right),
$$

which means that

$$
\widehat{H F S}(M, \gamma)=\widehat{H F S}\left(M_{2}, \gamma_{2}\right)
$$

by definition.

\subsection{An application to satellite knots}

As an application, we can compute the topmost terms in the knot Floer homology of satellite knots with nonzero winding numbers. We recall the following definition from [12].

Definition 5.8 Suppose $K$ is a null-homologous knot in $Y, F$ is a Seifert surface of $K$ (not necessarily of minimal genus). $V$ is a $3-$ manifold, $\partial V=T^{2}$, $L \subset V$ is a nontrivial knot. $G \subset V$ is a compact connected oriented surface so that $L$ is a component of $\partial G$, and $\partial G-L$ (may be empty) consists of parallel essential circles on $\partial V$. Orientations on these circles are induced from the orientation on $G$, we require that these circles are parallel as oriented ones. We glue $V$ to $Y-\operatorname{int}(\operatorname{Nd}(K))$, so that any component of $\partial G-L$ is null-homologous in $Y-\operatorname{int}(\operatorname{Nd}(K))$. The new manifold is denoted by $Y^{*}$, and the image of $L$ in $Y^{*}$ is denoted by $K^{*}$. We then say $K^{*}$ is a satellite knot of $K$, and $K$ a companion knot of $K^{*}$. Let $p$ denote the number of components of $\partial G-L, p$ will be called the winding number of $L$ in $V$.

Suppose $p>0, F$ is a minimal genus Seifert surface for $K$, then a minimal genus Seifert surface $F^{*}$ for $K^{*}$ can be obtained as follows: take $p$ parallel copies of $F$, and glue them to a certain surface $G$ in $V-\operatorname{int}(\operatorname{Nd}(L))$. We decompose $V-\operatorname{int}(\operatorname{Nd}(L))$ along $G$, the resulting sutured manifold is denoted by $(M(L), \gamma(L))$, where $\gamma(L)$ consists of $p+1$ annuli, $p$ of them lie on $\partial V$, denoted by $A_{1}, \ldots, A_{p}$. 
Corollary 5.9 With notations as above, suppose the genus of $K$ is $g>0$, and the genus of $K^{*}$ is $g^{*}$, then

$$
\widehat{H F K}\left(Y^{*}, K^{*},\left[F^{*}\right], g^{*}\right) \cong \widehat{H F K}(Y, K,[F], g) \otimes \widehat{H F S}(M(L), \gamma(L))
$$

as linear spaces over any field $\mathbb{F}$.

Proof Let $(M, \gamma)$ be the sutured manifold obtained by decomposing $Y^{*}-$ $\operatorname{int}\left(\operatorname{Nd}\left(K^{*}\right)\right)$ along $F^{*}$. Note that $A_{1}, \ldots, A_{p}$ are separating product annuli in $M$. The desired result holds by Theorem 5.1 .

Matthew Hedden also got some interesting results regarding knot Floer homology of satellite knots with nonzero winding numbers [8]. Our result can be compared with his.

\section{Characteristic product regions}

Definition 6.1 Suppose $(M, \gamma)$ is an irreducible sutured manifold, $\gamma$ has no toral component, $R_{-}(\gamma), R_{+}(\gamma)$ are incompressible and diffeomorphic to each other. A product region for $M$ is a submanifold $\Phi \times I$ of $N$, such that $\Phi$ is a compact, possibly disconnected, surface, and $\Phi \times 0, \Phi \times 1$ are incompressible subsurfaces of $R_{-}(\gamma), R_{+}(\gamma)$, respectively.

There exists a product region $E \times I$, such that if $\Phi \times I$ is any product region for $M$, then there is an ambient isotopy of $M$ which takes $\Phi \times I$ into $E \times I$. $E \times I$ is called a characteristic product region for $M$.

The theory of characteristic product regions is actually a part of JSJ theory ([9], [10]), the version that we need in the current paper can be found in [1].

The following theorem can be abbreviated as: if $\widehat{H F S}(M, \gamma) \cong \mathbb{Z}$, then the characteristic product region carries all the homology. A version of this theorem is also proved by Ian Agol via a different approach.

Theorem 6.2 Suppose $(M, \gamma)$ is an irreducible balanced sutured manifold, $\gamma$ has only one component, and $(M, \gamma)$ is vertically prime. Let $E \times I \subset M$ be the characteristic product region for $M$.

If $\widehat{H F S}(M, \gamma) \cong \mathbb{Z}$, then the map

$$
i_{*}: \mathrm{H}_{1}(E \times I) \rightarrow \mathrm{H}_{1}(M)
$$

is surjective. 
By Proposition 3.1, $(M, \gamma)$ is a homology product.

Let $G$ be a genus-1 compact surface with one boundary component. Glue the two sutured manifolds $(M, \gamma)$ and $G \times I$ together along their vertical boundaries, we get a sutured manifold $N$ with empty suture. $N$ has two boundary components $\Sigma=\Sigma_{-}=R_{-}(\gamma) \cup(G \times 0), \Sigma_{+}=R_{+}(\gamma) \cup(G \times 1) . N$ is also a homology product, thus there is a natural isomorphism

$$
\partial_{*}: \mathrm{H}_{2}(N, \partial N) \rightarrow \mathrm{H}_{1}(\Sigma) .
$$

Remark 6.3 Since $(M, \gamma)$ is a homology product, we can glue $R_{-}(\gamma)$ to $R_{+}(\gamma)$, so that the resulting manifold is the complement of a knot $K$ in a homology 3 -sphere $Y \not S^{3}$. Suppose $J$ is a genus-1 fibred knot in a homology sphere $Z \not S^{3}, G^{\prime}$ is a fibre. Consider the knot $K \# J \subset Y \# Z$. Let $Y_{0}^{\prime}$ be the manifold obtained by 0 -surgery on $K \# J$. Let $F=R_{-}(\gamma) \subset Y, H$ be the boundary connected sum of $F$ and $G^{\prime}$, and $\widehat{H}$ be the extension of $H$ in $Y_{0}^{\prime}$.

If we cut $Y_{0}^{\prime}$ open along $\widehat{H}$, then we get the manifold $N$. [4, Theorem 8.9] shows that $Y_{0}^{\prime}$ admits a taut foliation, with $\widehat{H}$ as a compact leaf. Thus this foliation induces a foliation of $N$.

Assume that the map

$$
i_{*}: \mathrm{H}_{1}(E \times I) \rightarrow \mathrm{H}_{1}(M)
$$

is not surjective. We can find a simple closed curve $\omega \subset R_{-}(\gamma)$, such that $[\omega]$ is not in $i_{*}\left(\mathrm{H}_{1}(E \times I)\right)$.

Let $\omega_{-}=\omega \subset \Sigma_{-}$, and let $\omega_{+} \subset \Sigma_{+}$be a circle homologous to $\omega$. We fix an arc $\delta$ connecting $\Sigma_{-}$to $\Sigma_{+}$. Let $\mathcal{S}_{m}(+\omega)$ be the set of properly embedded surfaces $S \subset N$, such that $\partial S=\omega_{-} \sqcup\left(-\omega_{+}\right)$, and the algebraic intersection number of $S$ with $\delta$ is $m$. Here $-\omega_{+}$denotes the curve $\omega_{+}$, but with opposite orientation. Similarly, let $\mathcal{S}_{m}(-\omega)$ be the set of properly embedded surfaces $S \subset N$, such that $\partial S=\left(-\omega_{-}\right) \sqcup \omega_{+}$, and the algebraic intersection number of $S$ with $\delta$ is $m$. Let $x\left(\mathcal{S}_{m}( \pm \omega)\right)$ be the minimal value of $x(S)$ for all $S \in \mathcal{S}_{m}( \pm \omega)$. It is obvious that

$$
x\left(\mathcal{S}_{m+1}( \pm \omega)\right) \leq x\left(\mathcal{S}_{m}( \pm \omega)\right)+x(\Sigma) .
$$

The next fact is implicitly contained in [2, Theorem 3.13].

Lemma 6.4 When $m$ is sufficiently large, there exist connected surfaces $S_{1} \in$ $\mathcal{S}_{m}(+\omega)$ and $S_{2} \in \mathcal{S}_{m}(-\omega)$, such that they give taut decompositions of $N$. 
Proof Let $D(N)$ be the double of $N$ along $\partial N . a=\partial_{*}^{-1}([\omega]) \in \mathrm{H}_{2}(N, \partial N)$ is the homology class whose intersection with $\Sigma$ is $[\omega], D(a)$ is its double in $\mathrm{H}_{2}(D(N))$. There exists $C \geq 0$, such that if $k>C$, then $x(D(a)+(k+1)[\Sigma])=$ $x(D(a)+k[\Sigma])+x(\Sigma)$. As in the proof of [2, Theorem 3.13], if $Q$ is a Thurston norm minimizing surface in the homology class $D(a)+k[\Sigma]$, and $Q \cap N$ has no disk or sphere components, then $Q \cap N$ gives a taut decomposition of $N$.

We can do oriented cut-and-paste of $Q$ with copies of $\Sigma$, to get a new surface $Q^{\prime}$, such that $Q^{\prime} \cap N$ has positive intersection number with $\delta$. Of course, $Q^{\prime} \cap N$ still gives a taut decomposition of $N$. The not-so-good thing is that $\partial\left(Q^{\prime} \cap N\right)$ is not necessarily $\omega_{-} \sqcup\left(-\omega_{+}\right)$. What we can do is to apply [3, Lemma 0.6]. Note that in the proof of [3, Lemma 0.6], one gets a new decomposition surface with prescribed boundary by gluing subsurfaces $W_{i}$ of $\Sigma_{ \pm}$to the original decomposition surface. And by [2, Lemma 3.10], $W_{i}$ has the same orientation as $\Sigma_{ \pm}$. So the algebraic intersection number of this new decomposition surface with $\delta$ is no less than $\left(Q^{\prime} \cap N\right) \cdot \delta>0$.

Denote the new decomposition surface by $S_{0}, \partial S_{0}=\omega_{-} \sqcup\left(-\omega_{+}\right)$. Suppose $S_{1}$ is the component of $S_{0}$ which contains $\omega_{-}$. For homological reason, $S_{1}$ should also contain $-\omega_{+}$. Thus other components of $S_{0}$ are closed surfaces which do not separate $\Sigma_{-}$from $\Sigma_{+}$. Hence the algebraic intersection number of other components with $\delta$ is $0 . S_{1}$ also gives a taut decomposition of $N$, by [3. Lemma 0.4]. So $S_{1}$ is the surface we need. Similarly, we can prove the result for $\mathcal{S}_{m}(-\omega)$.

We also need the following key lemma.

Lemma 6.5 For any positive integers $p, q$,

$$
x\left(\mathcal{S}_{p}(+\omega)\right)+x\left(\mathcal{S}_{q}(-\omega)\right)>(p+q) x(\Sigma) .
$$

Suppose $S_{1} \in \mathcal{S}_{p}(+\omega), S_{2} \in \mathcal{S}_{q}(-\omega)$. Isotope $S_{1}, S_{2}$ so that they are transverse. Since $N$ is irreducible and $S_{1}, S_{2}$ are incompressible, we can assume $S_{1} \cup S_{2}-$ $S_{1} \cap S_{2}$ has no disk components. Perform oriented cut-and-paste to $S_{1}, S_{2}$, we get a closed surface $P \subset \operatorname{int}(N)$, with $x(P)=x\left(S_{1}\right)+x\left(S_{2}\right)$. $P$ has no sphere components, otherwise $S_{1} \cup S_{2}-S_{1} \cap S_{2}$ would have disk components.

Now we will deal with the possible toral components of $P$. To this end, we need the following lemma.

Lemma 6.6 If $T \subset \operatorname{int}(N)$ is a torus, then the algebraic intersection number of $T$ and $\delta$ is 0 . 

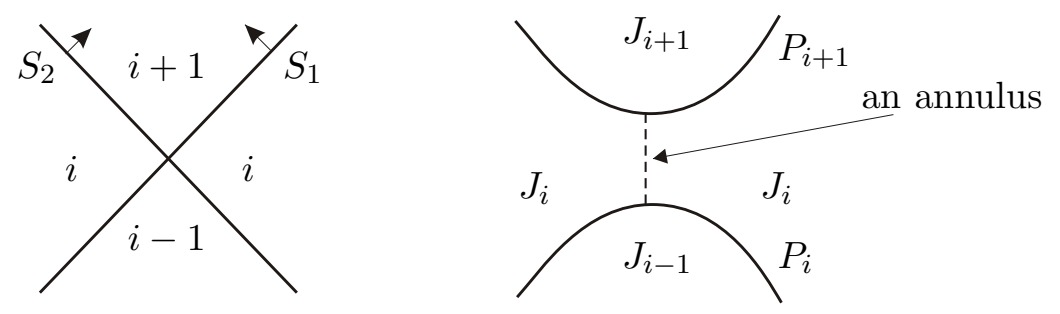

Figure 9: Doing oriented cut-and-paste to $S_{1}, S_{2}$.

Proof Since $N$ is a homology product, we have

$$
\mathrm{H}_{2}(D(N)) \cong \mathrm{H}_{2}(\Sigma) \oplus \mathrm{H}_{1}(\Sigma) \text {. }
$$

$T$ is disjoint from $\Sigma$, so $[T]$ must be a multiple of $[\Sigma]$ in $\mathrm{H}_{2}(D(N))$. By Remark 6.3, $\Sigma$ is Thurston norm minimizing. Since $x(\Sigma)>0=x(T)$, we must have $[T]=0$. Hence $T \cdot \delta=0$.

Suppose $T$ is a toral component of $P$, then $T$ is the union of $2 m$ annuli $A_{1}, A_{2}, \ldots, A_{2 m}$, where $A_{2 i-1} \subset S_{1}, A_{2 i} \subset S_{2}$. Let

$$
\begin{aligned}
& S_{1}^{\prime}=\left(S_{1}-\bigcup_{i=1}^{m} A_{2 i-1}\right) \cup \bigcup_{i=1}^{m}\left(-A_{2 i}\right) \\
& S_{2}^{\prime}=\left(S_{2}-\bigcup_{i=1}^{m} A_{2 i}\right) \cup \bigcup_{i=1}^{m}\left(-A_{2 i-1}\right) .
\end{aligned}
$$

Here $-A_{j}$ means $A_{j}$ with opposite orientation.

A small isotopy will arrange that $\left|S_{1}^{\prime} \cap S_{2}^{\prime}\right|<\left|S_{1} \cap S_{2}\right|$. Moreover, $x\left(S_{1}^{\prime}\right)=x\left(S_{1}\right)$, $x\left(S_{2}^{\prime}\right)=x\left(S_{2}\right)$. We want to show that $S_{1}^{\prime} \in \mathcal{S}_{p}(\omega), S_{2}^{\prime} \in \mathcal{S}_{q}(-\omega)$. Obviously, $\partial S_{1}^{\prime}=\partial S_{1}=\omega_{-} \sqcup\left(-\omega_{+}\right), \partial S_{2}^{\prime}=\partial S_{2}=\left(-\omega_{-}\right) \sqcup \omega_{+}$. Lemma 6.6 shows that $S_{1}^{\prime} \cdot \delta=S_{1} \cdot \delta$. Thus $S_{1}^{\prime} \in \mathcal{S}_{p}(+\omega)$. Similarly, $S_{2}^{\prime} \in \mathcal{S}_{q}(-\omega)$. Therefore, we can replace $S_{1}, S_{2}$ with $S_{1}^{\prime}, S_{2}^{\prime}$, then continue our argument.

Now we can assume $P$ has no toral components, and proceed to the proof of Lemma 6.5. Our approach to this lemma was suggested by David Gabai. In fact, this argument is similar to the argument in [4, Lemma 8.22].

Proof of Lemma 6.5 If $x\left(\mathcal{S}_{p}(+\omega)\right)+x\left(\mathcal{S}_{q}(-\omega)\right) \leq(p+q) x(\Sigma)$, then we can get a surface $P \subset \operatorname{int}(N)$ as above, $x(P) \leq(p+q) x(\Sigma)$. Define a function 
$\varphi:(N-P) \rightarrow \mathbb{Z}$ as follows. When $z \in \Sigma_{-}, \varphi(z)=0$. In general, given $z \in N-P$, choose a path from $\Sigma_{-}$to $z, \varphi$ is defined to be the algebraic intersection number of this path with $P$.

$N$ has the homology type of $\Sigma$, thus any closed curve in $N$ should have zero algebraic intersection number with any closed surface. Thus $\varphi$ is well-defined. Moreover, the value of $\varphi$ on $\Sigma_{+}$is $p+q$.

Let $J_{i}$ be the closure of $\{x \in(N-P) \mid \varphi(x)=i\}, P_{i}=J_{i-1} \cap J_{i}$. Thus $P=\sqcup_{i=1}^{p+q} P_{i}$, and $\cup_{k=0}^{i-1} J_{k}$ gives a homology between $\Sigma$ and $P_{i}$. Since $x(P) \leq$ $(p+q) x(\Sigma)$, and $\Sigma$ is Thurston norm minimizing in $D(N)$, we must have $x\left(P_{i}\right)=x(\Sigma)$ for each $i$.

$P_{i}$ has only one component. Otherwise, suppose $P_{i}=Q_{1} \sqcup Q_{2}$, then

$$
x\left(Q_{1}\right), x\left(Q_{2}\right)<x\left(P_{i}\right)=x(\Sigma) .
$$

As in the proof of Lemma 6.6, we find that $\left[Q_{1}\right],\left[Q_{2}\right]$ are multiples of $[\Sigma]$, which gives a contradiction.

We can isotope $P$, so that $P_{i} \cap M$ is a genus $g$ surface with one boundary component. In fact, after an isotopy, we can arrange that $P \cap \gamma_{1}$ consists of parallel essential curves in $\gamma_{1}$. Since $F$ and $G$ are Thurston norm minimizing in $\mathrm{H}_{2}(M, \gamma)$ and $\mathrm{H}_{2}(G \times I, \partial G \times I)$, respectively, we must have

$$
x\left(P_{i} \cap M\right)=x(F), x\left(P_{i} \cap(G \times I)\right)=x(G)=1 .
$$

If an annulus $A$ is a component of $P_{i} \cap(G \times I)$, then we can isotope $A$ inside $G \times I$ into $\partial G \times I$, a further isotopy of $P$ will decrease the number of components of $P \cap(G \times I)$. So we can assume that $P_{i} \cap(G \times I)$ has no annular components. Now the fact that $x\left(P_{i} \cap(G \times I)\right)=1$ indicates that $P_{i} \cap(G \times I)$ is either a thrice-punctured sphere or a once-punctured torus. In the former case there would be an essential sphere in $\widehat{G} \times I$, where $\widehat{G}$ is the torus obtained by capping off $\partial G$, which is impossible. Hence $P_{i} \cap(G \times I)$ is a once-puncture torus, and $P_{i} \cap M$ is diffeomorphic to $F$.

Since $M$ is vertically prime, $P_{i} \cap M$ is parallel to either $R_{-}(\gamma)$ or $R_{+}(\gamma)$ in $M$. Now the picture of $P_{1}, \ldots, P_{p+q}$ is clear: there exists a number $r \in$ $\{0,1, \ldots, p+q\}$, such that $P_{1}, \ldots, P_{r}$ are parallel to $\Sigma_{-}$, and $P_{r+1}, \ldots, P_{p+q}$ are parallel to $\Sigma_{+}$. Here we let $P_{0}=\Sigma_{-}, P_{p+q+1}=\Sigma_{+}$.

Since $P$ is gotten by doing cut-and-paste to $S_{1}, S_{2}$, we can isotope $S_{1}$ so that $S_{1} \cap J_{i}$ consists of vertical annuli. See Figure 9 for the local picture. We denote $S_{1} \cap J_{i}$ by $C_{i} \times I$, where $C_{i}$ is the collection of some circles in $P_{i}$. Obviously, $\left[C_{i}\right]$ is homologous to $[\omega]$. 
Consider $J_{r}$, which is bounded by $P_{r}, P_{r+1} . J_{r}$ is homeomorphic to $N$. Since

$$
[\omega] \notin \operatorname{im}\left(i_{*}: \mathrm{H}_{1}(E \times I) \rightarrow \mathrm{H}_{1}(N)\right),
$$

one component of $C_{r} \times I$ must lie outside $\operatorname{im} i_{*}$. Thus this vertical annulus can not be homotoped into $E \times I$, which contradicts to the definition of characteristic product region.

Lemma 6.7 Let $K$ be a knot in a homology 3-sphere $Y, Y_{p}$ be the manifold obtained by $p$-surgery on $K$. Let $g>1$ be the genus of $K$. Suppose $\widehat{H F K}(Y, K, g ; \mathbb{Q}) \cong \mathbb{Q}$, then

$$
H F^{+}\left(Y_{0},[g-1] ; \mathbb{Q}\right) \cong \mathbb{Q} .
$$

Proof We will use $\mathbb{Q}$ coefficients in the homologies. As in [13, Corollary 4.5], when $p$ is sufficiently large, we have two exact triangles

$$
\begin{aligned}
& \stackrel{\delta}{\longrightarrow} \widehat{H F K}(Y, K, g) \stackrel{\sigma}{\longrightarrow} H F^{+}\left(Y_{p},[g-1]\right) \stackrel{\psi}{\longrightarrow} H F^{+}(Y) \stackrel{\delta}{\longrightarrow}, \\
& \stackrel{\delta^{\prime}}{\longrightarrow} H F^{+}\left(Y_{0},[g-1]\right) \stackrel{\sigma^{\prime}}{\longrightarrow} H F^{+}\left(Y_{p},[g-1]\right) \stackrel{f}{\longrightarrow} H F^{+}(Y) \stackrel{\delta^{\prime}}{\longrightarrow} .
\end{aligned}
$$

And $f$ has the form $\psi+\iota$, where $\iota$ is a sum of homogeneous maps which have lower orders than $\psi$.

Since $\widehat{H F K}(Y, K, g ; \mathbb{Q}) \cong \mathbb{Q}$, either $\delta$ is surjective or $\sigma$ is injective. Therefore, either $\psi$ is injective, or $\psi$ is surjective. For simplicity, denote $H F^{+}\left(Y_{p},[g-1]\right)$ by $A$, and $H F^{+}(Y)$ by $B$.

If $\psi$ is injective, $B$ can be written as $\psi(A) \oplus C$ for some subgroup $C$ of $B$. If $b \in B$ is in the form of $(\psi(a), c)$, then let $\rho(b)=a$. Thus $\rho$ is a homomorphism, $\rho \psi=\mathrm{id}$, and $\iota \rho: B \rightarrow B$ is a homomorphism which strictly decreases degree. Now

$$
\mathrm{id}-\iota \rho+(\iota \rho)^{2}-(\iota \rho)^{3}+\cdots
$$

is a well-defined homomorphism. and

$$
\psi=\left(\mathrm{id}-\iota \rho+(\iota \rho)^{2}-(\iota \rho)^{3}+\cdots\right)(\psi+\iota) .
$$

Hence $f=\psi+\iota$ is also injective, and $H F^{+}\left(Y_{0},[g-1]\right) \cong B / f(A)$.

It is easy to check that id $+\iota \rho$ induces a homomorphism from $B / \psi(A)$ to $B / f(A)$, whose inverse is induced by

$$
\text { id }-\iota \rho+(\iota \rho)^{2}-(\iota \rho)^{3}+\cdots .
$$

Thus $B / f(A) \cong B / \psi(A)$. So $\operatorname{rank}\left(H F^{+}\left(Y_{0},[g-1]\right)\right)=1$.

A similar argument shows that if $\psi$ is surjective, then $f$ is also surjective, and $\operatorname{rank}\left(H F^{+}\left(Y_{0},[g-1]\right)\right)=1$. 
Proof of Theorem 6.2 Use the notations in Remark 6.3, we have

$$
\operatorname{rank}(\widehat{H F K}(Y \# Z, K \# J, g+1))=\operatorname{rank}(\widehat{H F K}(Y, K, g))=1 .
$$

$\operatorname{Thus} \operatorname{rank}\left(H F^{+}\left(Y_{0}^{\prime}, g\right)\right)=1$ by Lemma 6.7

If $i_{*}$ is not surjective, then the proof of [5, Theorem 1.4], combined with Lemma 6.4 and Lemma 6.5, shows that

$$
\operatorname{rank}\left(H F^{+}\left(Y_{0}^{\prime}, g\right)\right)>1
$$

which gives a contradiction.

More precisely, by Lemma 6.4 and Lemma 6.5, there exist connected surfaces $S_{1} \in \mathcal{S}_{m}(+\omega)$ and $S_{2} \in \mathcal{S}_{m}(-\omega)$, such that they give taut decompositions of $N$, and $x\left(S_{1}\right)+x\left(S_{2}\right)>2 m x(\Sigma)$. By Gabai's work [2, Section 5], there exist two taut foliations $\mathscr{F}_{1}, \mathscr{F}_{2}$ of $N$, such that

$$
\begin{aligned}
& \chi\left(S_{1}\right)=e\left(\mathscr{F}_{1}, S_{1}\right)=e\left(\mathscr{F}_{1}, S_{0}\right)+m \chi(\Sigma), \\
& \chi\left(S_{2}\right)=e\left(\mathscr{F}_{2}, S_{2}\right)=e\left(\mathscr{F}_{2},-S_{0}\right)+m \chi(\Sigma) .
\end{aligned}
$$

Here $e(\mathscr{F}, S)$ is defined in [5, Definition 3.7], $S_{0}$ is any surface in $\mathcal{S}_{0}(+\omega)$.

Now we can conclude that $e\left(\mathscr{F}_{1}, S_{0}\right) \neq e\left(\mathscr{F}_{2}, S_{0}\right)$. Hence [5, Theorem 3.8] can be applied.

\section{Proof of the main theorem}

Proof of Theorem 1.1 Suppose $(M, \gamma)$ is the sutured manifold obtained by cutting open $Y-\operatorname{int}(\operatorname{Nd}(K))$ along $F, E \times I$ is the characteristic product region. We need to show that $M$ is a product. By Proposition 3.1, $M$ is a homology product. Moreover, by Theorem 4.1, we can assume $M$ is vertically prime.

If $M$ is not a product, then $M-E \times I$ is nonempty. Thus there exist some product annuli in $(M, \gamma)$, which split off $E \times I$ from $M$. Let $\left(M^{\prime}, \gamma^{\prime}\right)$ be the remaining sutured manifold. By Theorem 6.2, $R_{ \pm}\left(\gamma^{\prime}\right)$ are planar surfaces, and $M^{\prime} \cap(E \times I)$ consists of separating product annuli in $M$. Since we assume that $M$ is vertically prime, $M^{\prime}$ must be connected. (See the first paragraph in the proof of Theorem 5.1.) Moreover, $M^{\prime}$ is also vertically prime, and there are no nontrivial product disks or product annuli in $M^{\prime}$. By Theorem 5.1, $\widehat{H F S}\left(M^{\prime}, \gamma^{\prime}\right) \cong \mathbb{Z}$.

We add some product 1 -handles to $M^{\prime}$ to get a new sutured manifold $\left(M^{\prime \prime}, \gamma^{\prime \prime}\right)$ with $\gamma^{\prime \prime}$ connected. By Proposition 2.9 , $\widehat{H F S}\left(M^{\prime \prime}, \gamma^{\prime \prime}\right) \cong \mathbb{Z}$. It is easy to see 
that $M^{\prime \prime}$ is also vertically prime. Proposition 3.1 shows that $M^{\prime \prime}$ is a homology product.

In the manifold $M^{\prime \prime}$, the characteristic product region $E^{\prime \prime} \times I$ is the union of the product 1 -handles and $\mathrm{Nd}\left(\gamma^{\prime}\right)$. Obviously $i_{*}: \mathrm{H}_{1}\left(E^{\prime \prime}\right) \rightarrow \mathrm{H}_{1}\left(M^{\prime \prime}\right)$ is not surjective, which contradicts to Theorem 6.2.

Proof of Corollary 1.2 Cut $Y-\operatorname{int}(\operatorname{Nd}(L))$ open along $F$, we get a sutured manifold $(M, \gamma), \widehat{H F S}(M, \gamma) \cong \mathbb{Z}$. By adding product 1 -handles with feet at $\gamma$, we can get a new sutured manifold $\left(M^{\prime}, \gamma^{\prime}\right)$, where $\gamma^{\prime}$ has only one component. We have $\widehat{H F S}\left(M^{\prime}, \gamma^{\prime}\right) \cong \mathbb{Z}$. By Theorem 1.1, $M^{\prime}$ is a product, hence $M$ is also a product. So the desired result holds.

\section{References}

[1] D Cooper, D Long, Virtually Haken Dehn-filling, J. Differential Geom. 52 (1999), no. 1, 173-187

[2] D Gabai, Foliations and the topology of 3-manifolds, J. Differential Geom. 18 (1983), no. 3, 445-503

[3] D Gabai, Foliations and the topology of 3-manifolds II, J. Differential Geom. 26 (1987), no. 3, 461-478

[4] D Gabai, Foliations and the topology of 3-manifolds III, J. Differential Geom. 26 (1987), no. 3, 479-536

[5] P Ghiggini, Knot Floer homology detects genus-one fibred knots, preprint (2006), arXiv:math.GT/0603445

[6] H Goda, Heegaard splitting for sutured manifolds and Murasugi sum, Osaka J. Math. 29 (1992), no. 1, 21-40

[7] E Grigsby, Knot Floer Homology in Cyclic Branched Covers, Algebr. Geom. Topol. 6 (2006), 1355-1398 (electronic)

[8] M Hedden, private communication

[9] W Jaco, P Shalen, Seifert fibered spaces in 3-manifolds, Mem. Amer. Math. Soc. 21 (1979), no. 220

[10] K Johannson, Homotopy equivalences of 3-manifolds with boundaries, Lecture Notes in Mathematics, 761. Springer, Berlin, 1979

[11] A Juhász, Holomorphic discs and sutured manifolds, Algebr. Geom. Topol. 6 (2006), 1429-1457 (electronic)

[12] Y Ni, Sutured Heegaard diagrams for knots, Algebr. Geom. Topol. 6 (2006), 513-537 (electronic) 
[13] P Ozsváth, Z Szabó, Holomorphic disks and knot invariants, Adv. Math. 186 (2004), no. 1, 58-116

[14] P Ozsváth, Z Szabó, Holomorphic disks and genus bounds, Geom. Topol. 8 (2004), 311-334 (electronic)

[15] P Ozsváth, Z Szabó, On knot Floer homology and lens space surgeries, Topology 44 (2005), no. 6, 1281-1300

[16] P Ozsváth, Z Szabó, Heegaard Floer homologies and contact structures, Duke Math. J. 129 (2005), no. 1, 39-61

[17] P Ozsváth, Z Szabó, Knot Floer homology and rational surgeries, preprint, available at arXiv:math.GT/0504404

[18] P Ozsváth, Z Szabó, Heegaard diagrams and holomorphic disks, Different faces of geometry, 301-348, Int. Math. Ser. (N. Y.), Kluwer/Plenum, New York, 2004

[19] J Rasmussen, Floer homology and knot complements, PhD Thesis, Harvard University (2003), available at arXiv:math.GT/0306378

[20] J Stallings, On fibering certain 3-manifolds, Topology of 3-manifolds and related topics (Proc. The Univ. of Georgia Institute, 1961) pp. 95-100, PrenticeHall, Englewood Cliffs, New Jersey, 1962

[21] W Thurston, A norm for the homology of 3-manifolds, Mem. Amer. Math. Soc. 59 (1986), no. 339, i-vi and 99-130 